\title{
Density Functional Tight Binding Calculations for Probing Electronic-Excited States of Large Systems
}

\author{
Sharma S. R. K. C. Yamijala ${ }^{1}$, M. Belén Oviedo ${ }^{2}$, and Bryan M. Wong ${ }^{1}$ \\ ${ }^{1}$ Department of Chemical \& Environmental Engineering, Materials Science $\mathcal{E}$ \\ Engineering Program, Department of Chemistry, and Department of Physics 8 \\ Astronomy, University of California-Riverside, Riverside, California 92521, \\ United States \\ ${ }^{2}$ Departamento de Química Teórica y Computacional, Facultad de Ciencias \\ Químicas, Universidad Nacional de Córdoba, Instituto de Investigaciones en \\ Fisicoquímica de Córdoba (INFIQC), UNC-CONICET, Córdoba X5000HUA,
} Argentina

\section{Introduction}

Over the past decade, researchers in computational chemistry have witnessed a resurgence in the development and application of semi-empirical methods for treating large chemical/material systems. In particular, the density functional tight binding (DFTB) formalism ${ }^{1-4}$ has garnered immense popularity for probing the electronic properties of biomolecules, ${ }^{5}$ molecules/clusters with numerous conformations, ${ }^{6,7}$ and immense nanostructures. ${ }^{8}$ While classical molecular dynamics can handle hundreds of thousands of atoms, it cannot provide a first-principles-based description of large systems at a quantum mechanical (i.e., electronic) level of detail. At the other extreme, conventional 
Kohn-Sham density functional theory (DFT) methods can access the true quantum mechanical nature of matter, but they cannot tackle the large sizes and complex chemical environments relevant to many of the large systems mentioned previously. To bridge these immense size scales, the DFTB formalism was developed to probe these chemical/materials systems with a viable approach that is both computationally efficient and quantum mechanical in nature.

While there have been a number of good reviews on DFTB,${ }^{9-12}$ in this review focuses on the application of DFTB to electronic-excited states, which has attracted significant attention for extending this computationally efficient approach to the time-domain. Although the field of chemical dynamics is incredibly vast, we concentrate our attention on the application of DFTB to real-time electron dynamics and non-adiabatic dynamics calculations. We first give a brief discussion of the underlying theory in each of these areas, followed by a didactic tutorial on simple molecules or model systems to show how these computational approaches and techniques are carried out in practice. These simple tutorials are written in a stepwise, instructive fashion to provide practicing researchers a detailed "look under the hood" to obtain a deeper understanding of how these techniques can be used to understand, probe, and even control the chemical dynamics of large systems. We then conclude each of these respective sections with a "real-life" application of these DFTB-based approaches on large dynamical systems to demonstrate their usefulness in contemporary areas of chemistry and materials science. 


\section{REAL-TIME TIME-DEPENDENT DFTB (RT-TDDFTB)}

\subsection{Theory and Methodology}

Over the past few years, the use of real-time time-dependent DFTB (RTTDDFTB) has attracted significant attention as a promising approach for extending the DFTB formalism to the non-equilibrium electron dynamics of extremely large chemical systems. As specific examples, this method has been used to calculate photo-injection dynamics in dye-sensitized $\mathrm{TiO}_{2}$ solar cells, ${ }^{13-15}$ optical properties of photosynthetic pigments, ${ }^{16,17}$ molecular aggregates, ${ }^{18}$ graphene nanoflakes, ${ }^{19}$ DNA intercalation complexes, ${ }^{20}$ manybody interactions in solvated nanodroplets, ${ }^{21}$ and excitation energy transfer

dynamics in plasmonic arrays. ${ }^{22,23}$ To carry out an RT-TDDFTB dynamics calculation, one must first compute the ground-state Hamiltonian, overlap matrix elements, and the initial single-electron density matrix within the self-consistent DFTB approach. The open-source DFTB + code $^{24}$ provides a practical way to obtain these quantities, and a more detailed description of ground-state DFTB can be found in previous publications. ${ }^{25,26}$

Once the ground-state Hamiltonian, overlap matrix elements, and the initial single-electron density matrix are calculated, they can be used as initial input conditions in subsequent real-time quantum dynamics calculations. These RT-TDDFTB quantum dynamics calculations are carried out in practice by applying a time-dependent electric field to the initial ground state density matrix, resulting in the Hamiltonian

$$
\hat{H}(t)=\hat{H}^{0}-E_{0}(t) \cdot \hat{\mu}(t),
$$

where $E_{0}(t)$ is the applied electric field, and $\hat{\mu}$ is the dipole moment operator. Because the Hamiltonian in Eq. (1) is explicitly time dependent, the density matrix, $\hat{\rho}$, evolves according to the Liouville-von Neumann equation 
of motion which, in the nonorthogonal-DFTB basis, is given by ${ }^{27}$

$$
\frac{\partial \hat{\rho}}{\partial t}=\frac{1}{i \hbar}\left(S^{-1} \cdot \hat{H}[\hat{\rho}] \cdot \hat{\rho}-\hat{\rho} \cdot \hat{H}[\hat{\rho}] \cdot S^{-1}\right)
$$

where $\hat{H}$ is the Hamiltonian matrix (which implicitly depends on the density matrix), $S^{-1}$ is the inverse of the overlap matrix, and $\hbar$ is Planck's constant.

Furthermore, because the quantum system is directly propagated in the time domain, one can choose $E_{0}(t)$ to have any time-dependent form. For example, if $E_{0}(t)$ is chosen to have the form of a Dirac delta function given by

$$
E_{\text {delta }}(t)=E_{0} \delta\left(t-t_{0}\right)
$$

this "kick" perturbation instantaneously changes the velocity field of the electrons and causes a time-varying dipole moment. As a result, the state of the system is no longer an eigenfunction of the Hamiltonian. In practice, $E_{\text {delta }}$ is implemented as a linearly polarized Gaussian-type perturbation or as a phase in the initial electron density ${ }^{28}$ given by:

$$
E_{\text {delta }}(t)=E_{0} \exp \left[-\frac{\left(t-t_{0}\right)^{2} w^{2}}{2}\right] \hat{n}
$$

where $\hat{n}$ is the polarization vector. The temporal evolution of the density matrix is carried out iteratively where the atomic charge on atom $\alpha$ is first calculated using the Mulliken approximation:

$$
q_{\alpha}=\sum_{\mu \in \alpha}[\rho(t) S]_{\mu \mu}
$$

Afterwards, the self-consistent charge Hamiltonian matrix is constructed as ${ }^{4}$

$$
H_{\mu \nu}=\left\langle\phi_{\mu}\left|\hat{H}^{0}\right| \phi_{\nu}\right\rangle+\frac{1}{2} S \sum_{\xi}\left(\gamma_{\alpha \xi}+\gamma_{\beta \xi}\right) \Delta q_{\xi},
$$

where $\phi_{\mu}$ are DFTB Slater-type orbital basis functions centered on the atomic 
sites, and $\gamma_{\alpha \xi}$ is a function of the inter-atomic separation and the Hubbard parameter $U .{ }^{29}$ Finally, the density matrix is updated using a three-point integration algorithm:

$$
\hat{\rho}\left(t_{0}+\Delta t\right)=\hat{\rho}\left(t_{0}-\Delta t\right)-2 \frac{i}{\hbar} \dot{\hat{\rho}} \Delta t+O\left[(\Delta t)^{2}\right]
$$

which is accurate to order $(\Delta t)^{2}$.

However, if one chooses $E_{0}(t)$ to take the form of a sinusoidal perturbation given by

$$
E_{\text {laser }}=E_{0} \sin (\omega t),
$$

it represents a continuous interaction of the system with monochromatic light (i.e., a laser) in the time domain, and this perturbation is added to the Hamiltonian in the iterative procedure:

$$
\hat{H}(t)=\hat{H}_{0}+\frac{1}{2}[S \hat{V}(t)+\hat{V}(t) S]
$$

where $\hat{V}(t)$ is set to $-E_{0} \sin (\omega t) \cdot \hat{\mu}(t)$ or the expression for the delta perturbation (Eq. (4)). It is worth mentioning that both of these different choices for the electric field give different but complementary viewpoints of quantum dynamics, as will be explained in both the tutorial and example sections that follow. To more easily understand this entire procedure, the following pseudocode flowchart shows the sequential steps for carrying out an RT-TDDFTB electron dynamics calculation. ${ }^{30}$

In summary, the RT-TDDFTB algorithm commences by reading the following files from a ground-state DFTB + calculation: the non-self-consistent Hamiltonian matrix $\left(H_{0}\right)$, the self-consistent Hamiltonian matrix $\left(H_{\mathrm{SCC}}\right)$, the single-electron density matrix in the molecular orbital representation $\left(\rho_{\mathrm{MO}}\right)$, and the overlap matrix $(S)$. Next, the inverse of the overlap matrix $\left(S^{-1}\right)$ is computed, and $H_{\mathrm{SCC}}$ is diagonalized. The diagonalization of this matrix gives the one-electron reduced density matrix of the ground state in the atomic 


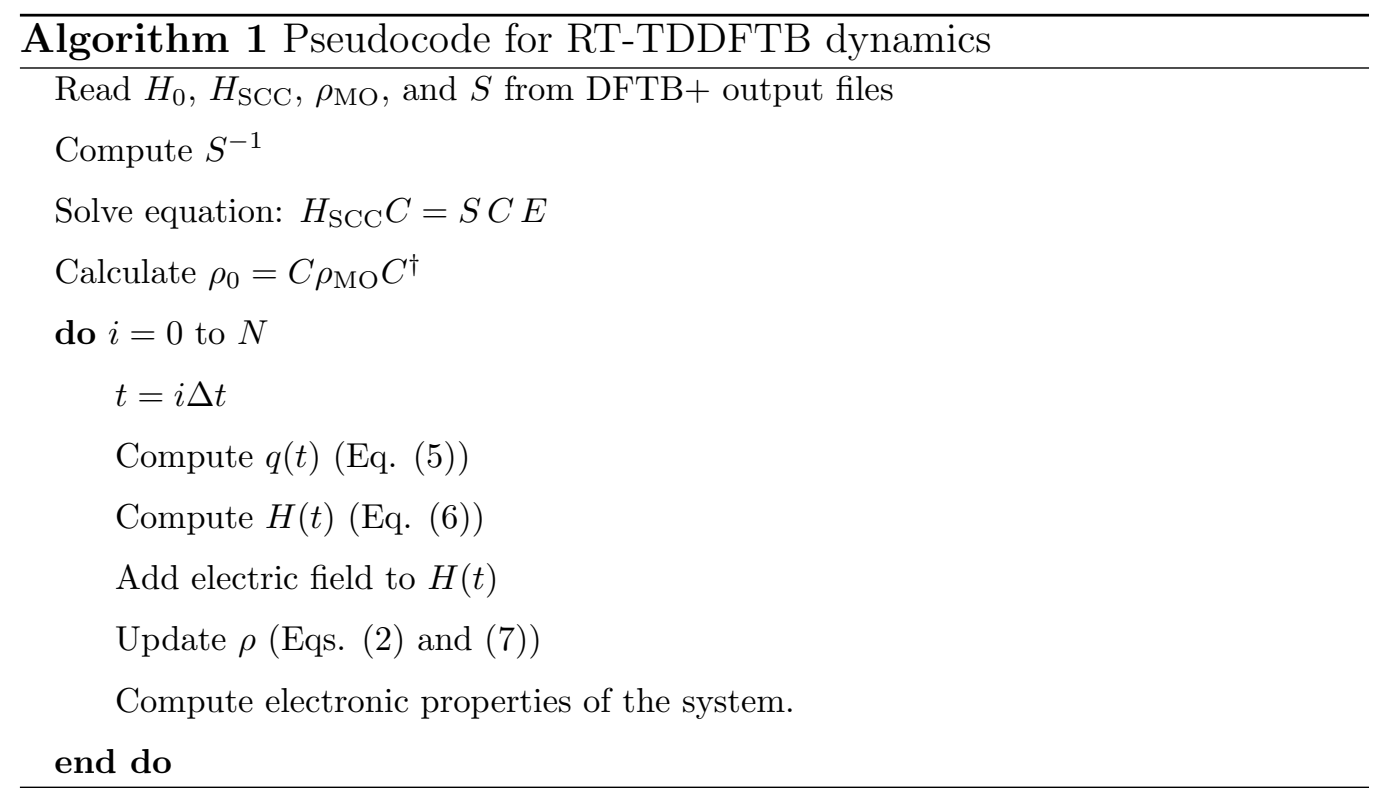

orbital basis.

\subsection{Tutorial on RT-TDDFTB Electron Dynamics for a Naphthalene Molecule}

With the basic theoretical concepts of RT-TDDFTB outlined in the previous section, we now give two tutorials showing how to (1) compute an absorption spectrum of a simple naphthalene molecule and (2) probe the time-dependent dynamics of naphthalene in the presence of monochromatic light (i.e., a laser perturbation) using the RT-TDDFTB approach.

\subsection{Absorption Spectrum for Naphthalene}

As mentioned in the RT-TDDFTB theory and methodology section, a groundstate DFTB + calculation for naphthalene must be carried out first before performing an RT-TDDFTB calculation. With the ground-state DFTB+ calculation for naphthalene properly converged, one obtains the non-self- 
consistent Hamiltonian matrix, the self-consistent Hamiltonian matrix, the initial single-electron density matrix, and the overlap matrix as output files. Next, to compute an absorption spectrum of naphthalene, 3 independent simulations must be carried out in which the system is excited with a very short electric pulse (i.e., a Dirac delta pulse) that is applied in 3 mutually orthogonal directions to compute the polarizability tensor. Once the RTTDDFTB electron dynamics calculation finishes, the time-dependent dipole moment is obtained, as shown in Fig. 1.

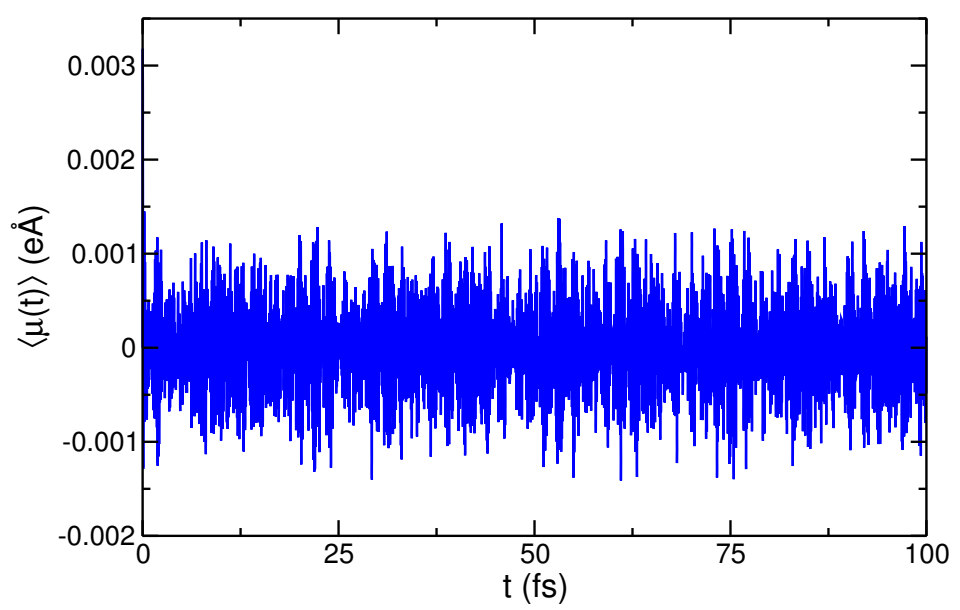

Figure 1: Time-dependent dipole moment for a naphthalene molecule obtained by applying a Dirac delta electric field pulse to the system.

In the limit of very weak perturbations, the system is said to be in the linear response regime, and the induced dipole moment of the system is given by

$$
\mu(t)=\int_{-\infty}^{\infty} \alpha(t-\tau) E(\tau) d \tau
$$

where $\tau$ is the time difference between the electric field and induced dipole moment, $E$ is the electric field used to induce a rearrangement of charges inside the system, and $\alpha$ is the polarizability tensor. The quantity most easily accessible experimentally is the photo-absorption cross section given 
by:

$$
\sigma(\omega)=\frac{4 \pi \omega}{c} \operatorname{Im}(\alpha)
$$

where $c$ is the speed of light, and $\operatorname{Im}(\alpha)$ is the imaginary part of the average polarizability. $\operatorname{Im}(\alpha)$ is obtained by the application of the convolution theorem, and Eq. (10) can be expressed in the frequency domain as

$$
\mu(\omega)=\alpha(\omega) E(\omega)
$$

To mimic the experimental absorption spectrum within this formalism, a damping term is typically included in Eq. (12). This effectively incorporates a finite lifetime of the excited state in the response function, which produces a broadening in the absorption spectrum peaks ${ }^{31,32}$. The damping factor used in this tutorial on naphthalene is $0.01 \mathrm{fs}^{-1}$ (see left panel of Fig. 2). This approach gives the polarizability along the direction of the initially applied field, and the full polarizability tensor is obtained from three independent time propagations where the only quantity that is altered is the direction of the external electric field. ${ }^{33}$ The average of the polarizability along the three Cartesian axes is taken as the absorption spectrum of naphthalene, as shown in the right-hand side of Fig. 2.
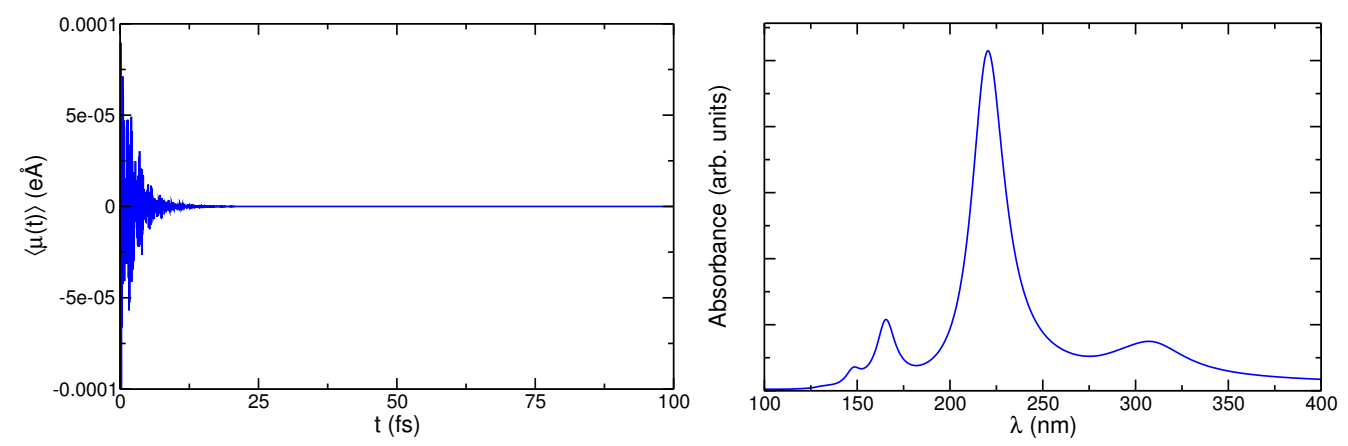

Figure 2: (left) Damped time-dependent dipole moment for a naphthalene molecule, and (right) absorption spectrum for naphthalene obtained from the Fourier transform of the damped time-dependent dipole moment. 


\subsection{Electron Dynamics of Naphthalene with a Laser- Type Perturbation}

With the absorption spectrum of naphthalene properly computed, one can apply a laser-type perturbation tuned to the lowest excitation energy (i.e., $5.63 \mathrm{eV}$ ) of the system. As in the case of the Dirac delta pulse, one can also set the polarization of the laser field to any orientation with respect to the molecule; however, for this example on naphthalene, we have oriented the laser field in the direction of maximum polarizability to produce the maximum variation of the dipole moment as a function of time, as shown in Fig. 3.

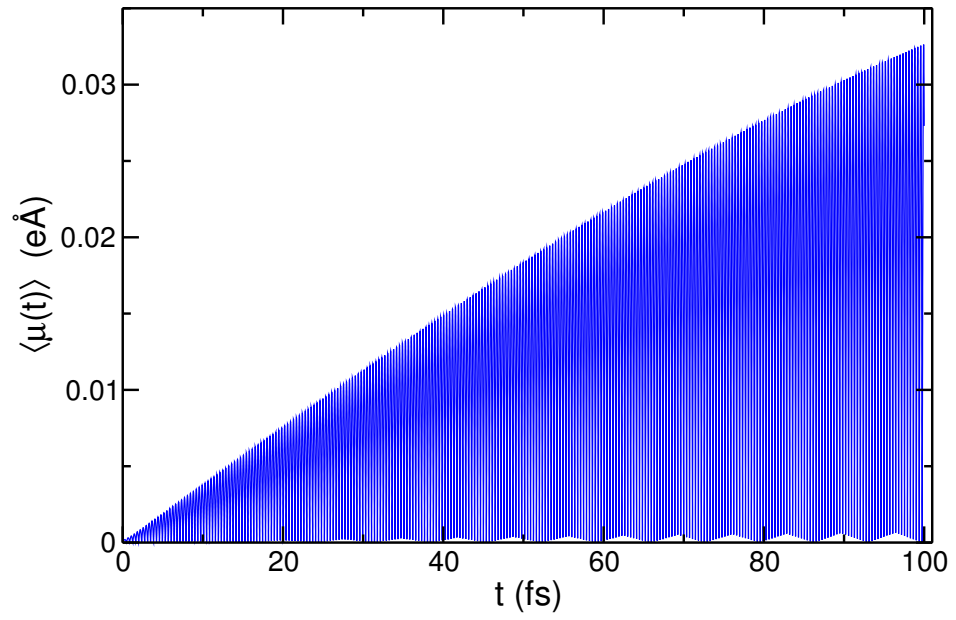

Figure 3: Time-dependent dipole moment for a naphthalene molecule obtained by applying a laser field tuned to the lowest excitation energy (5.63 $\mathrm{eV}$ ) of the system.

It can be noted from Fig. 3 that the naphthalene molecule exhibits a linearly increasing dipole moment, as expected from a quantized system in the linear response regime that is continuously excited and in the absence of any dissipative mechanisms. ${ }^{34}$ As such, this can be used to check that the laser is in tune with the electronic excitation energy and that the simulation is indeed in the linear response regime. 


\subsection{RT-TDDFTB Electron Dynamics of a Realistic Large Systems}

In this final section on RT-TDDFTB dynamics, we give an example of the techniques used in the previous tutorial section on a "real-life" application to a large system. In particular, we focus on the real-time electron dynamics of excitation energy transfer (EET) in a large plasmonic nanoantenna system using the RT-TDDFTB formalism. ${ }^{22,23}$ Understanding and achieving a controlled transfer of energy in these novel systems has been a continual area of interest in various technologies including nanophotonic circuits, ${ }^{35-38}$ waveguide materials, ${ }^{39-41}$ and other natural light-harvesting antenna systems. ${ }^{42,43}$

\section{Absorption Spectrum of a Single Plasmonic Nanoparti- cle}

We begin our analysis of EET by first characterizing the plasmon resonance energy of a single nanoparticle (NP) containing 55 silver atoms and having an icosahedral shape. The geometry of this NP was optimized with the DFTB+ package using the hyb-0-2 set of DFTB parameters (available at dftb.org), and its absorption spectrum was obtained in the same manner as described in the tutorial section on naphthalene. As can be seen in Fig. 4, a prominent peak, corresponding to the plasmon resonance is observed around $3.23 \mathrm{eV}$. This result is in good agreement with a time-dependent density functional theory (DFT) calculation of $3.6 \mathrm{eV}^{44}$ and a recent experimental result of 3.8 $\mathrm{eV}^{45}$ for similar-sized $\mathrm{Ag}$ nanoparticles.

\section{Exploring Excitation Energy Transfer in Ag Nanopar- ticle Chains}

With the energy of a single Ag nanoparticle characterized, we can now proceed to an analysis of EET in plasmonic NP assemblies. Accordingly, we can 


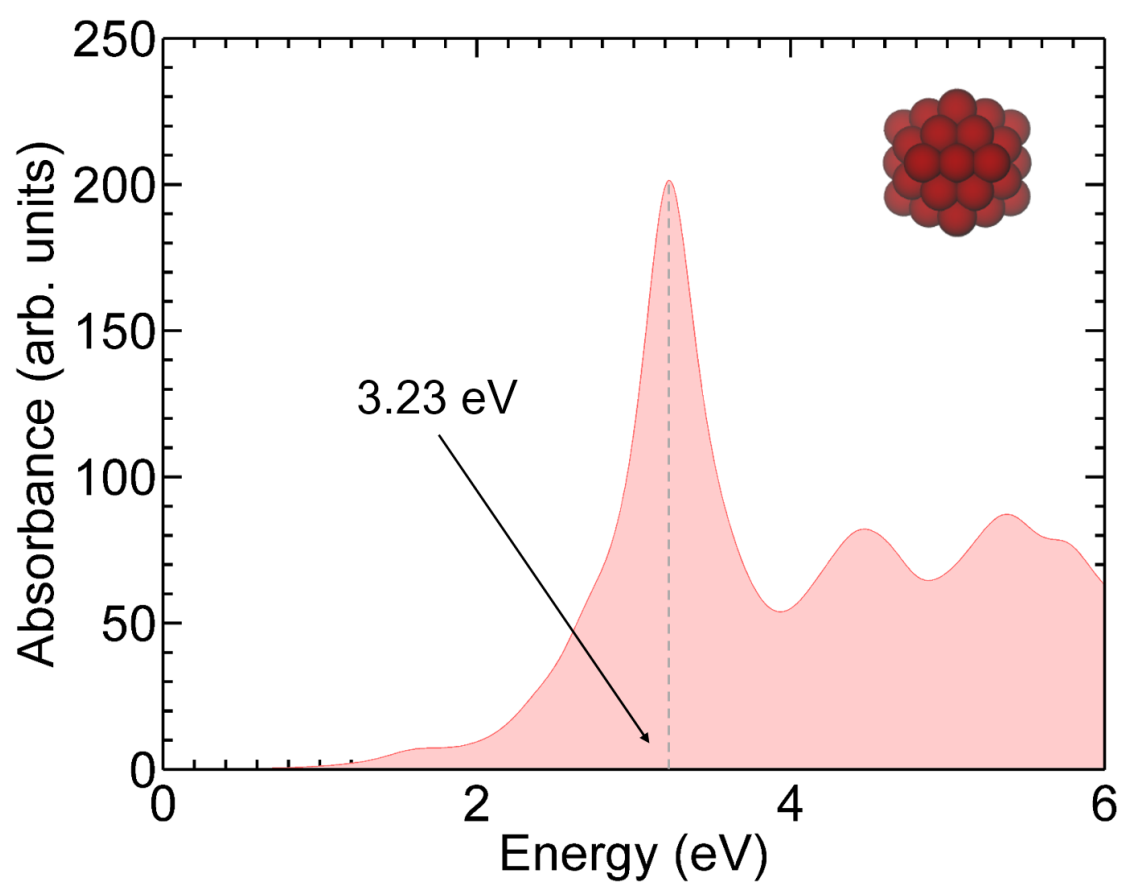

Figure 4: Absorption spectrum of a 55 atom icosahedral silver nanoparticle. A prominent plasmon resonance peak is observed around $3.23 \mathrm{eV}$. Source: from Ref. 23 with permission from The Royal Society of Chemistry.

use the single Ag NP to construct a variety of NP antenna configurations, each with interparticle distances $(d)$ varying from 5 to $0.5 \AA$. We define the interparticle distance as the edge-to-edge distance between the NPs, and two of the model NP waveguides are shown in Fig. 5. We can also construct a NP chain where the NPs "touch" each other $(d=0 \AA)$ in which the centerto-center distance between two atoms from adjacent NPs is less than the $\mathrm{Ag}-\mathrm{Ag}$ bond-forming distance (the $\mathrm{Ag}-\mathrm{Ag}$ atom bond length is $3.00 \AA$ ). Note that each of these chains are extremely large systems containing a total of 440 atoms and, therefore, would be computationally prohibitive to calculate with conventional real-time TDDFT approaches.

To simulate EET along the NP chains, we excite only the first Ag NP in 
(a)

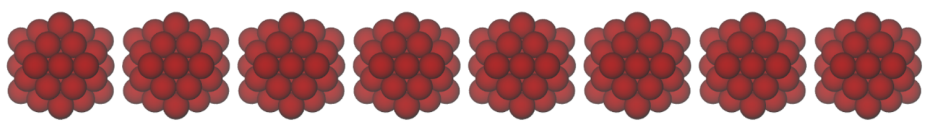

(b)

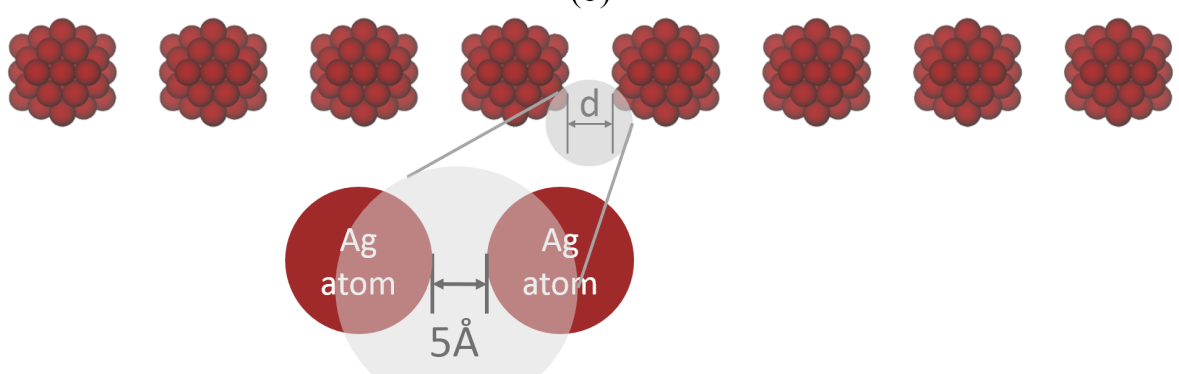

Figure 5: Pictorial representation of two finite chains with $8 \mathrm{Ag}$ NPs of radius $\approx 1.23 \mathrm{~nm}$ and interparticle (edge-to-edge) distances equal to (a) $1 \AA$ and (b) $5 \AA$. Source: from Ref. 23 with permission from The Royal Society of Chemistry.

the chain using a monochromatic laser (using a similar approach discussed in the tutorial section) with an energy equal to the plasmonic resonance energy of a single Ag NP (3.23 eV). With this chosen initial condition, the entire system is allowed to evolve in time according to Eq. (2). To quantify the EET efficiency along the chain, we can compute the electric field intensities, $I=\sqrt{\epsilon_{0} / \mu_{0}} \times|\mathbf{E}|^{2}$, at identical points between each of the NPs along the axial direction shown in Fig. 6. $\mathbf{E}$ is the total electric field, and $\epsilon_{0}$ and $\mu_{0}$ are the permittivity and permeability of free space, respectively.

Fig. 7 shows the intensity trends of the NP chains with interparticle distances ranging from 0 to $5 \AA$. From the intensity trends in Fig. 7, we observe a monotonic increase in the EET efficiency (i.e., the slope of the intensity lines decreases) as the interparticle distance is reduced from 5 to about $2 \AA$. This result is in qualitative agreement with previous studies on similar systems using classical electrodynamic methods. ${ }^{46,47}$ This increase 


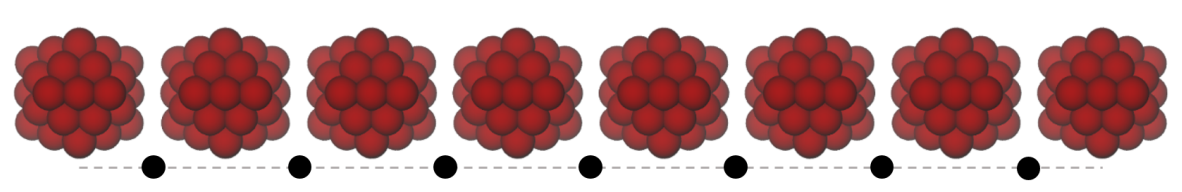

Figure 6: The field intensity values are taken at identical positions in each nanoparticle chain as shown by the black dots. The points lie exactly between two nanoparticles and on a line approximately $1 \AA$ below the lowest atom in the NP. Source: from Ref. 23 with permission from The Royal Society of Chemistry.

in EET efficiency can be attributed to an increase in capacitive coupling between the Ag NPs as the interparticle distance between them is reduced. In other words, this phenomenon is analogous to a charged capacitor, ${ }^{48}$ where the capacitance of a capacitor increases as the charged plates are brought closer together. However, as the interparticle distance is further reduced below $2 \AA$, we observe an opposite trend of the EET efficiency. In particular, we see a sudden drop in EET efficiency for interparticle distances below $2 \AA$ (i.e., the slope of the intensity line increases). This result is qualitatively opposite to what has been predicted by previous computational studies that have observed a decrease in EET when the NPs directly touch each other. ${ }^{46,47}$

\section{Analyzing the Electronic Couplings in NP Chains}

To understand these interesting dynamical effects, we can use RT-TDDFTB to analyze in detail the electronic couplings between the NPs in the plasmonic chain. To this end, we plot the RT-TDDFTB absorption spectra of Ag NP dimers with varying interparticle distances in Fig. 8. On careful observation of Fig. 8, we note that a single prominent peak, close to the value of the single NP plasmonic energy, can be observed for all interparticle distances. However, for interparticle spacings less than $2 \AA$, an additional peak (marked with arrows in Fig. 8) forms in the absorption spectrum. The prominent 


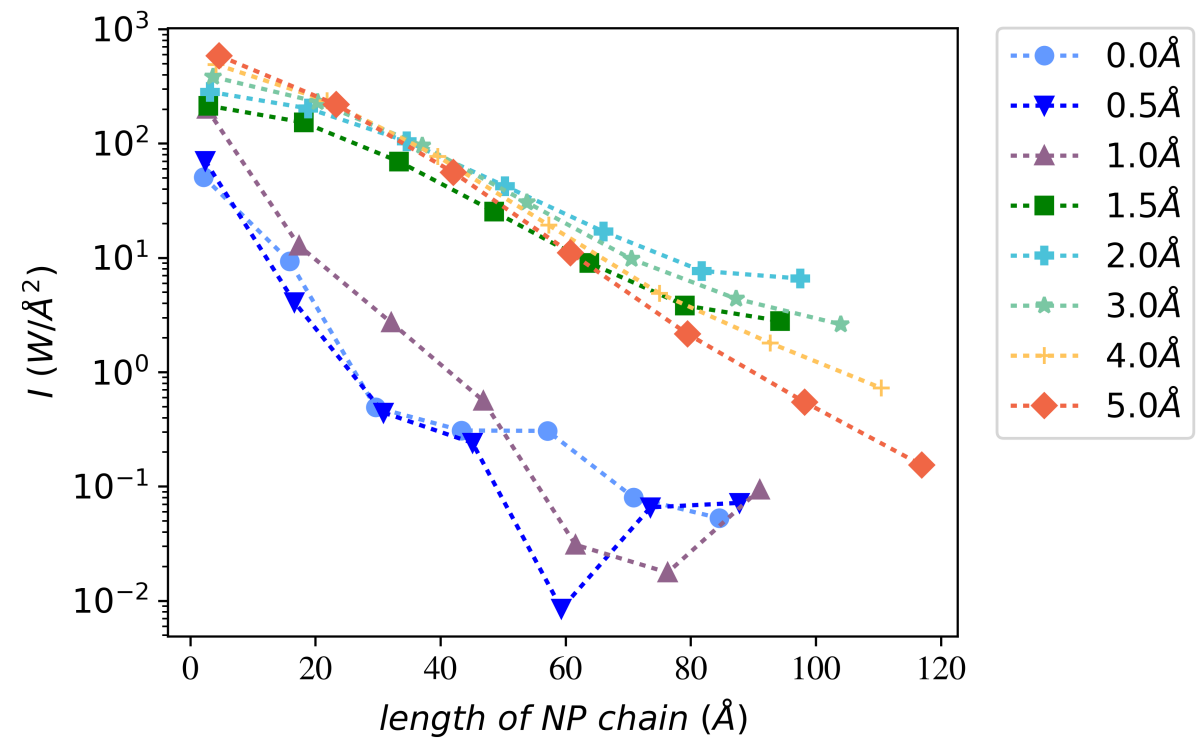

Figure 7: Field intensities along silver NP chains with varying interparticle distances. The first nanoparticle in each of the chains is excited at the plasmon resonance energy, and the intensity values are computed at the interparticle gaps of the NPs as shown in Figure 6. The excitation energy used in the simulation is equal to the plasmon resonance energy of the single $\mathrm{Ag}$ nanoparticle. A drastic drop in the field intensity is seen for Ag chains with interparticle spacings less than $2 \AA$. Source: from Ref. 23 with permission from The Royal Society of Chemistry.

peak normally arises due to interactions (hybridizations) between the basic plasmon resonances of the elementary nanostructures (in this case, the single Ag NP). This excitation is the bonding (symmetric) mode, normally known as the Bonding Dipole Plasmon, or BDP, and is characterized by charge oscillations of the NPs in phase with each other. ${ }^{49}$ The other peak appearing at lower energies and smaller interparticle distances is normally observed when an optical-frequency conductive pathway is established between two NPs, enabling the transfer of charge between them. This conductive pathway can be physical, due to a physical bridge or due to quantum tunneling. This 
is known as a Charge Transfer Plasmon, or CTP. ${ }^{50}$ Unlike the BDP, the CTP is characterized by a total charge moving between the two nanoparticles of the dimer, which we observe as the lower-energy peak in our absorption spectrum. In our case of non-touching NPs, the CTP excitation can be attributed completely to quantum tunneling that establishes a conductive pathway between the two NPs of the dimer. While charge transfer plasmons have been previously observed theoretically in DFT and quantum-corrected classical models, ${ }^{48,50,51}$ this is the first example of predicting CTPs using RT-TDDFTB calculations.

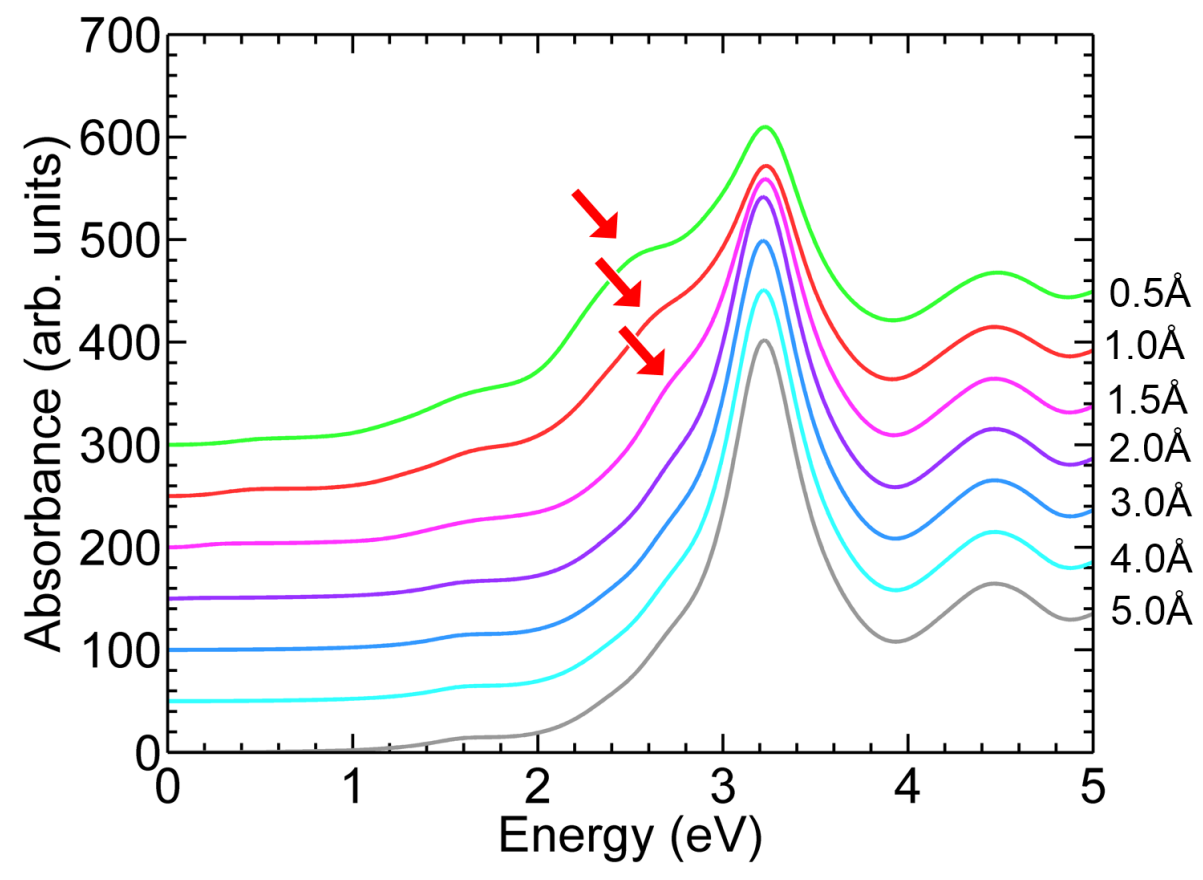

Figure 8: Absorption spectrum for Ag NP dimers with varying interparticle separations. An additional lower-energy peak (corresponding to a charge transfer plasmon excitation) emerges in the absorption spectrum for dimers having an interparticle spacing less than $2 \AA$, denoted by arrows. Source: from Ref. 23 with permission from The Royal Society of Chemistry. 


\section{Investigating the Nature of Plasmonic Excitations}

To understand these drastic drops in EET efficiency for smaller interparticle spacings, we need an intuitive way to analyze these excitations. Fig. 9 shows the charge distributions and the time-dependent changes in Mulliken charges for the NP dimer with an interparticle spacing of $1 \AA$. In the panels of this figure, we compare the time-dependent dynamics when the NP dimer is excited at either the BDP or CTP energy peak. When the NP dimer is excited at the CTP peak, one of the NPs shows a predominantly positive charge, while the other one shows a negative charge (Fig. 9(a)). The time-dependent changes in Mulliken charges confirm this observation in Fig. 9(c). This behavior is characteristic of a CTP excitation, where an oscillating current occurs between the two NPs of the dimer. Also note in Fig. 9(a) that we observe a slight dipolar nature of charge distributions near the particle edges. This can be attributed to the atomistic treatment of the nanoparticles, whereby the charge transfer plasmon induced on the nanoparticle dimers also establishes a small opposing dipole on the inner edges of the same nanoparticles due to inter-atomic electrodynamic interactions.

However, when the NP is excited at the BDP peak, we observe some charge transfer from one NP to the other, which is uncharacteristic of a BDP excitation. ${ }^{22}$ In particular, we observe that at subnanometer interparticle spacings, the pure BDP excitation forms a hybridized excitation that has some CTP character. As such, the decrease in the EET efficiency in smaller interparticle spacing chains can be attributed to the formation of this hybridized BDP. Because the hybridized BDP allows for a small charge transfer between the NPs, it reduces the capacitive coupling between the NPs. Going back to the capacitor analogy used previously, this can be thought of as a leaking capacitor. This, in turn, is ultimately responsible for the reduction in capacitive coupling between the NPs and hence the drop in EET efficiency, as revealed by these RT-TDDFTB calculations. 
(a)

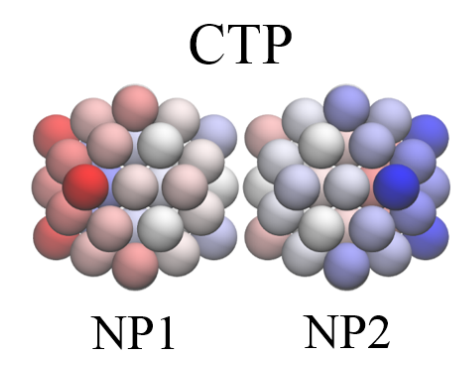

(c)

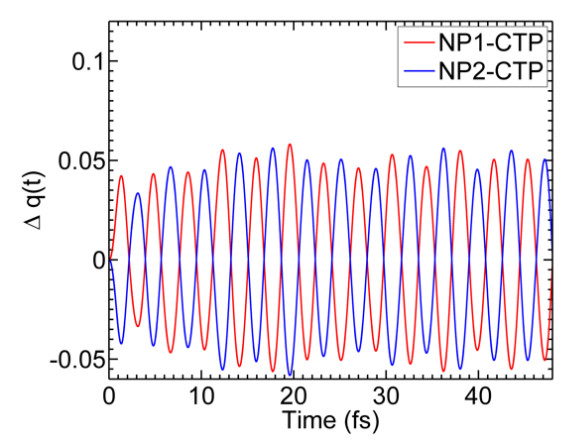

(b)

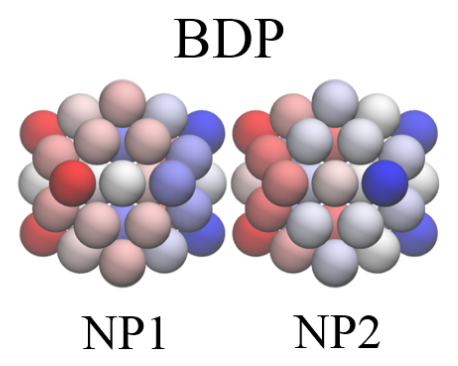

(d)

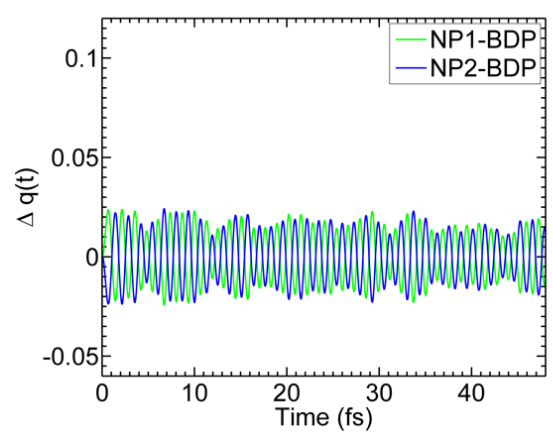

Figure 9: Snapshot of charge distributions at one instance in time for a $\mathrm{Ag}$ NP dimer with an interparticle distance equal to $1 \AA$ excited at (a) the CTP peak and (b) the BDP peak. The CTP peak distributions show a total charge separation between the two NPs, while the BDP peak distributions show dipolar charge distributions within each of the NPs. The time-dependent changes in Mulliken charges are shown for the (c) CTP and (d) BDP peak for the same Ag NP dimer. For both the CTP and the BDP excitations, a net charge fluctuation is seen between the NPs, which indicates a hybridized nature of the BDP peak at subnanometer spacings. Source: from Ref. 23 with permission from The Royal Society of Chemistry. 


\section{DFTB-BASED NONADIABATIC ELEC- TRON DYNAMICS}

\subsection{Adiabatic Vs. Nonadiabatic Dynamics}

Until now, we have highlighted the use of RT-TDDFTB to probe the electron dynamics of large systems in external electric fields where the nuclei are held fixed. However, in this section, we discuss and give examples where this constraint is relaxed, and the nuclei are allowed to evolve nonadiabatically on different potential surfaces. We first give a general overview of nonadiabatic dynamics and present a specific example of how DFTB can be further extended to give mechanistic insight in these excited-state processes.

In conventional electronic structure methods (such as DFT or even DFTB), one can solve the time-independent Schrödinger equation (TISE), $\hat{H} \Phi=\epsilon \Phi$, for a given set of nuclear coordinates $(\mathbf{R})$. Specifically, the electronic Hamiltonian is diagonalized to obtain a set of eigenvectors and eigenvalues that depend on the nuclear coordinates and, therefore, are known as the adiabatic eigenvectors and eigenvalues. When the TISE is solved for several sets of nuclear coordinates, we obtain the adiabatic potential energy surfaces (PESs).

In many chemical reactions, the wavefunction of the entire system can be expressed as a single adiabatic potential energy surface (instead of a linear combination of several adiabatic surfaces). Often, this adiabatic PES corresponds to the ground-state of the species involved in the chemical reaction. For example, when we draw a reaction path depicting the transformation of the reactants $\left(\mathrm{HCl}+\mathrm{C}_{2} \mathrm{H}_{5} \mathrm{Br}\right)$ into the products $\left(\mathrm{HBr}+\mathrm{C}_{2} \mathrm{H}_{5} \mathrm{Cl}\right)$ through a transition state $\left(\mathrm{C}_{2} \mathrm{H}_{5} \mathrm{BrCl}\right)$, we inherently assume that these reactions occur along the ground-state PES (through a specific reaction coordinate). These chemical processes, where the wavefunction is confined to a single PES, are termed as adiabatic processes (see left panel of Fig. 10) and can be accurately described by the TISE (i.e., when the nuclear dynamics 
are governed by a single adiabatic PES, it is within the realm of the adiabatic dynamics). Born-Oppenheimer molecular dynamics and Car-Parrinello molecular dynamics are examples of such ab-initio adiabatic dynamics, and many electronic structure packages such as CP2K, ${ }^{52}$ Quantum Espresso, ${ }^{53}$ VASP,${ }^{54}$ SIESTA, ${ }^{55}$ NWChem, ${ }^{56}$ and DFTB $+{ }^{57}$ are routinely used by many researchers to calculate on-the-fly adiabatic dynamics.

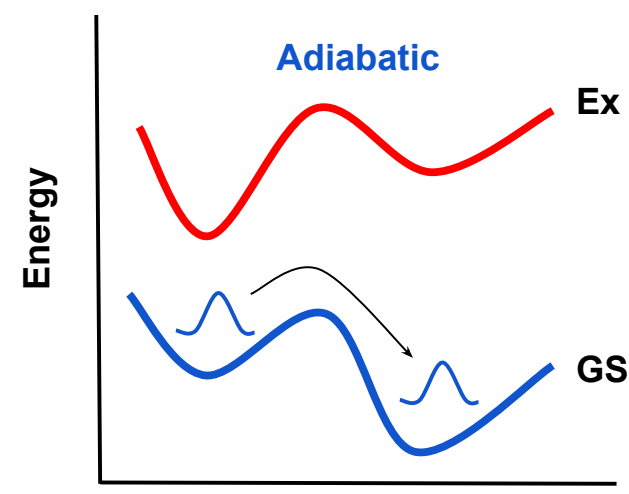

Reaction Coordinate

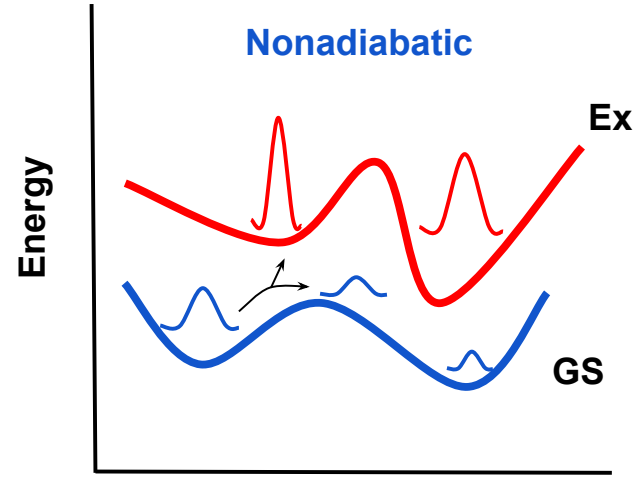

Reaction Coordinate

Figure 10: In an adiabatic processes (left panel), the wavefunction of the entire system is confined to a single PES, typically the ground-state (GS) surfacce. In a nonadiabatic process (right panel), the electronic amplitudes of the wavefunction will evolve over many PESs (i.e., the wavefunction is expressed as a linear combination of several adiabatic states).

In contrast to adiabatic dynamics, in most photochemical processes, the wavefunction of the entire system spans several PESs. A well-known example of this is fluorescence (i.e., the decay of a system from an excited (Ex) to the ground state (GS) by emitting radiation), and the wavefunction of the entire system cannot be solely described as either the ground or excited state, but can only be expressed as a superposition of these states. These processes, where the electronic amplitudes of the wavefunction evolve over many PESs, are known as nonadiabatic processes (right panel of Fig. 10). Furthermore, the nuclear motion that is described by several adiabatic PESs is known as nonadiabatic molecular dynamics (NAMD). Compared to adiabatic dynam- 
ics, only a few software packages such as Q-Chem ${ }^{58}$ and Octopus ${ }^{33}$ have implemented direct on-the-fly nonadiabatic molecular dynamics (i.e., the entire NAMD simulation can be performed within the same package). Although direct on-the-fly implementations are scarce, many software packages such as PYXAID, ${ }^{59}$ NEWTON-X, ${ }^{60}$ SHARC,${ }^{61}$ QMFlows, ${ }^{62}$ and LIBRA ${ }^{63}$ have implemented various NAMD methods. Also, most of these packages have an interface with traditional electronic structure packages such as Quantum Espresso and others, enabling us to perform NAMD calculations on both molecular and periodic systems. In this review, we present a tutorial and example of the Fewest-Switches Surface Hopping method (a NAMD method), which we implemented in the DFTB+ software package.

To accurately capture nonadiabatic effects, a full quantum mechanical treatment of both the electronic and nuclear degrees of freedom is necessary. However, such a treatment is computationally prohibitive even for systems with moderate sizes ( $\sim 50-100$ atoms). To circumvent this computational burden, only the electrons are treated quantum mechanically, and the nuclear motion is described classically. Among these so-called "mixed quantumclassical" approaches, the Fewest-Switches Surface-Hopping (FSSH) method is one of the most popular methods, and in this review, we use it to study the nonadiabatic electron dynamics in organic systems.

\subsection{Equations Governing Nonadiabatic Electron Dy- namics}

In any mixed quantum-classical (MQC) method, the electron dynamics is captured by a time-dependent total wavefunction, $\Psi(\mathbf{r}, t)$, which satisfies the time-dependent electronic Schrödinger equation (TDSE):

$$
i \hbar \frac{\partial}{\partial t}|\Psi(\mathbf{r}, t)\rangle=\hat{H}_{\mathrm{el}}(\mathbf{r}, \mathbf{R}(t))|\Psi(\mathbf{r}, t)\rangle .
$$

Here, $\hat{H}_{\mathrm{el}}(\mathbf{r}, \mathbf{R}(t))$ is the electronic Hamiltonian operator for a set of nu- 
clear coordinates, $\mathbf{R}$, at time $t$. Using an electronic structure method such as DFT, the electronic Hamiltonian can be solved to obtain the adiabatic eigenvectors $\left.\Phi_{i}(\mathbf{r}, \mathbf{R}(t))\right\rangle$ and eigenvalues $\epsilon_{i}(\mathbf{R}(t))$. These adiabatic eigenvectors can correspond to molecular orbitals, Slater determinants, etc., depending on the employed electronic structure method. Accordingly, the corresponding eigenvalues can represent the molecular orbital energies, ground or excited state energies, etc.

By assuming that these adiabatic eigenvectors form a complete orthonormal basis, we can express the total wavefunction as their linear combination, i.e.,

$$
|\Psi(\mathbf{r}, t)\rangle=\sum_{i} a_{i}(t)\left|\Phi_{i}(\mathbf{r}, \mathbf{R}(t))\right\rangle .
$$

Inserting the above ansatz (Eq. (14)) into the TDSE (Eq. (13)), and multiplying the resulting expression with $\left\langle\Phi_{j}(\mathbf{r}, \mathbf{R}(t))\right|$ from the left gives us the following differential equation:

$$
\dot{a}_{j}(t)=-\frac{i}{\hbar} a_{j}(t) \epsilon_{j}(\mathbf{R}(t))-\sum_{i} a_{i}(t)\left\langle\Phi_{j}(t) \mid \frac{\partial}{\partial t} \Phi_{i}(t)\right\rangle .
$$

In this expression we have used the shorthand notation $\left|\Phi_{i}(t)\right\rangle \equiv\left|\Phi_{i}(\mathbf{r}, \mathbf{R}(t))\right\rangle$, and the non-adiabatic coupling elements, $C_{j i} \equiv\left\langle\Phi_{j}(t) \mid \frac{\partial}{\partial t} \Phi_{i}(t)\right\rangle$ are related to the derivative coupling vectors, $\mathbf{d}_{j i}$, through

$$
C_{j i}=\dot{\mathbf{R}}\left\langle\Phi_{j}(\mathbf{r}, \mathbf{R}(t))\left|\nabla_{\mathbf{R}}\right| \Phi_{i}(\mathbf{r}, \mathbf{R}(t))\right\rangle \equiv \dot{\mathbf{R}} \mathbf{d}_{j i} .
$$

It is important to note that the coupling elements, $C_{j i}$, are responsible for the nonadiabatic electronic transitions between any two adiabatic states $j$ and $i$. Integrating Eq. (15) gives us the wavefunction coefficients, $a_{j}(t)$, at each time-step, allowing us to completely specify the total wavefunction, $|\Psi(\mathbf{r}, t)\rangle$ (we already know the adiabatic eigenfunctions at each nuclear step by solving the TISE). As a reminder, the set of $\left\{\left|\Phi_{p}(\mathbf{r}, \mathbf{R}(t))\right\rangle\right\}$ is known as an adiabatic basis because it explicitly depends on the nuclear positions, $\mathbf{R}$. 


\subsection{The Classical Path Approximation}

As mentioned earlier, in MQC methods, the nuclei are propagated according to classical mechanics, and the forces acting on the nuclei at each nuclear time-step can be obtained using the Hellmann-Feynman theorem:

$$
F_{I}=-\left\langle\Psi(\mathbf{r}, t)\left|\nabla_{I} \hat{H}_{\mathrm{el}}(\mathbf{r}, \mathbf{R}(t))\right| \Psi(\mathbf{r}, t)\right\rangle .
$$

Thus, the changes in the total wavefunction with time will have a direct influence on the nuclear motion. Often, to decrease computational costs, the electronic "back reaction" on the nuclear motion is neglected, and this approximation is known as the classical path approximation (CPA). As such, within the CPA, the nuclear motion is not affected by the electron dynamics. Nonetheless, the electron dynamics still depends on the nuclear coordinates (due to the parametric dependence of the adiabatic eigenvectors on the nuclear coordinates at each time-step), and the CPA is generally valid when the ground and excited state PESs differ slightly.

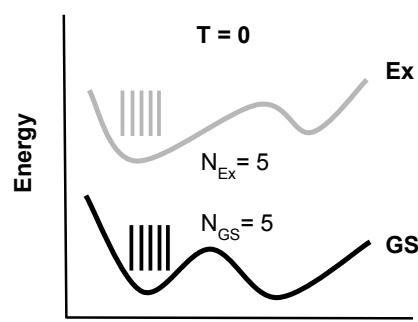

(a)

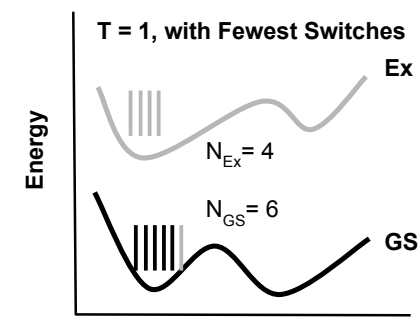

(b)

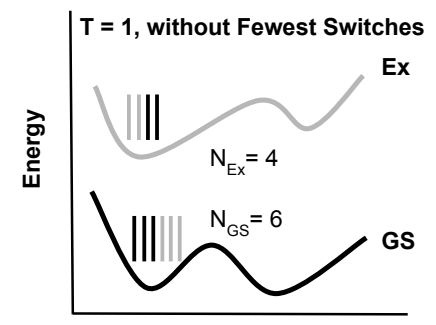

(c)

Reaction Coordinate

Figure 11: Number of trajectories in the excited $\left(N_{\mathrm{Ex}}\right)$ and ground state $\left(N_{\mathrm{GS}}\right)$ at times (a) $t=0$, (b) $t=1$, satisfying the fewest switches criterion, and (c) $t=1$, without satisfying the fewest switches criterion. Trajectories that are present in the excited (ground) state at time $t=0$ are shown in gray (black) to emphasize the fewest switches criterion at time $t=1$. 


\subsection{Surface Hopping and Fewest Switches Criterion}

Surface hopping is a general MQC nonadiabatic dynamics methodology with many variants, such as Fewest Switches Surface Hopping (FSSH), ${ }^{64,65}$ Decoherence Induced Surface Hopping (DISH), ${ }^{66}$ Independent Electron Surface Hopping (IESH), ${ }^{67}$ and others. ${ }^{68}$ The similarities in most of these variants include the following:

1. The nuclei are propagated according to classical mechanics, and the forces on the nuclei, at any given instant of time, arise from a single adiabatic PES.

2. The nonadiabatic electron dynamics are captured by evolving the wavefunction using a stochastic algorithm for each trajectory and by averaging the results over a swarm of trajectories.

For each Surface Hopping (SH) trajectory, we start our simulation from a single adiabatic PES (known as the active state). Next, we compute the probabilities for an electron to hop from this active state to all the other states (i.e., the adiabatic PESs). These computed probabilities are then compared with a uniformly generated random number. If the probability to jump from the active state to any other state is greater than the generated random number, the hop is accepted; otherwise, it will be rejected. This process is repeated for a swarm of trajectories, and the results are averaged over them to obtain the electron dynamics (as further explained in the next section).

Among the variants of surface hopping approaches, the FSSH method is the most successful. ${ }^{59,68-71}$ In this method, the number of hops (switches) between the states is minimized, as described in the following example. Let us assume that our system has only two states or PESs (namely, ground and excited states), and we are running 10 surface hopping trajectories. Let us also consider that at a particular instant of time (say, $t=0$ ), 5 of these 10 trajectories are in the excited state, while the other 5 are in the ground state; 
i.e., $\left[N_{\mathrm{GS}}, N_{\mathrm{Ex}}\right]=[5,5]$ (see Fig. 11a). Finally, let us assume that in the next time step $(t=1)$, there are six trajectories in the ground state $\left(N_{\mathrm{GS}}=6\right)$, and four in the excited state $\left(N_{\mathrm{Ex}}=4\right)$. We can obtain this configuration, $\left[N_{\mathrm{GS}}, N_{\mathrm{Ex}}\right]=[6,4]$, in several ways including: (1) switching 3 trajectories from the excited state to the ground state, and only 2 trajectories from the ground to the excited state (Fig. 11c) or (2) allowing 2 trajectories to hop from the excited state to the ground state, and only 1 trajectory to hop from the ground to the excited state, and so on. There will always be a way to achieve the desired configuration with the least number of switches/hops between the potential energy surfaces. To achieve the desired configuration with minimum switches in the present example, only 1 trajectory in the excited state should switch to the ground state, and zero trajectories should switch from the ground state to the excited state (Fig. 11b). This criterion is known as the fewest-switches (FS) criterion, and using this constraint, Tully proposed the FSSH mechanism to capture nonadiabatic electron dynamics phenomena. We use the CPA version of FSSH in conjunction with DFTB (as the underlying electronic structure method), as explained below.

\subsection{Implementation Details of CPA-FSSH-DFTB}

To understand charge transfer dynamics in large organic photovoltaic systems, we use the CPA-FSSH method in the DFTB+ software package (version 17.1). In particular, we use the DFTB3 variant ${ }^{72}$ as our electronic structure method to obtain the adiabatic eigenvectors and eigenvalues at each nuclear time step. For this implementation, we assume that the photoinduced excited state dynamics can be well described with a single-electron wavefunction. Hence, we adapt the single particle version of the time-dependent Kohn-Sham (TDKS) approximation, ${ }^{73}$ which assumes the time-dependent excited state wavefunction $|\Psi(\mathbf{r}, t)\rangle$ can be represented as a linear combination of the ground state KS orbitals $\left|\Phi_{i}(\mathbf{r}, \mathbf{R}(t))\right\rangle$, as given in Eq. (14). This definition of $|\Psi(\mathbf{r}, t)\rangle$ has been shown to provide reasonable photoinduced 
charge transfer dynamics. ${ }^{74-78}$ Using the above definition, Eq. (15) can be numerically integrated using the fourth-order Runge-Kutta (RK4) method to obtain the expansion coefficients, $a_{i}(t)$, at each time step. During the integration, to compute the non-adiabatic coupling elements (Eq. (16)), we use the following well-established approximation ${ }^{64}$

$$
\left\langle\Phi_{j}(t) \mid \frac{\partial}{\partial t} \Phi_{i}(t)\right\rangle=\frac{1}{2 \tau}\left[\left\langle\Phi_{j}(t) \mid \Phi_{i}(t+\tau)\right\rangle-\left\langle\Phi_{j}(t+\tau) \mid \Phi_{i}(t)\right\rangle\right] .
$$

This quantity needs to be carefully calculated by following the random phases generated during the electronic structure calculations to obtain the adiabatic eigenvectors, $\left|\Phi_{i}(\mathbf{r}, \mathbf{R}(t))\right\rangle \cdot{ }^{79-81}$ In DFTB + , these eigenvectors (MOs) are expanded as a linear combination of the atomic orbitals (AOs); i.e.,

$$
\left|\Phi_{i}(\mathbf{r}, \mathbf{R}(t))\right\rangle=\sum_{\mu} C_{\mu i}(\mathbf{r}, \mathbf{R}(t))\left|\varphi_{\mu}(\mathbf{r}, \mathbf{R}(t))\right\rangle,
$$

and in Eq. (18), the overlap between the adiabatic basis at two different time steps is computed as

$$
\left\langle\Phi_{j}(\mathbf{r}, \mathbf{R}(t+\tau)) \mid \Phi_{i}(\mathbf{r}, \mathbf{R}(t))\right\rangle=\sum_{\mu \nu} C_{\mu j}(\mathbf{r}, \mathbf{R}(t+\tau)) C_{\nu i}(\mathbf{r}, \mathbf{R}(t)) S_{\mu \nu}(t+\tau, t) .
$$

Here, $S_{\mu \nu}(t+\tau, t)$ is the overlap between two atomic orbitals (AOs) at two different time steps

$$
S_{\mu \nu}(t+\tau, t)=\left\langle\varphi_{\mu}(\mathbf{r}, \mathbf{R}(t+\tau)) \mid \varphi_{\nu}(\mathbf{r}, \mathbf{R}(t))\right\rangle .
$$

These AO overlap integrals are explicitly evaluated with our in-house version of the DFTB+ code.

After obtaining the eigenvectors, eigenvalues, wavefunction expansion coefficients, and the coupling elements at each time step, the surface hopping simulation is carried out using the CPA-FSSH scheme proposed by Akimov 
and Prezhdo, ${ }^{59}$ which is an adaptation of the original FSSH scheme proposed by Tully and Hammes-Schiffer (with some modifications). ${ }^{64}$ In CPA-FSSH, an instantaneous active state is assigned and the probability of switching from the current state, $\left|\Phi_{i}(\mathbf{r}, \mathbf{R}(t))\right\rangle$, to any other state, $\left|\Phi_{j}(\mathbf{r}, \mathbf{R}(t))\right\rangle$, during a small time interval, $t \in[t, t+\delta]$ is calculated as

$$
\tilde{g}_{i j}(t)=-\frac{2 \operatorname{Re}\left[\rho_{i j}(t)\left\langle\Phi_{j}(t) \mid \frac{\partial}{\partial t} \Phi_{i}(t)\right\rangle\right]}{\rho_{i i}(t)} \delta t,
$$

where, $\rho_{i j}(t)=a_{i}^{*}(t) a_{j}(t)$ are the adiabatic electronic density matrix elements. Because of the classical path approximation, one can ignore the back reaction of the electronic-non-adiabatic transition on the nuclear degrees of freedom. Hence, we do not re-scale the velocity, which is one of the key ingredients in the original FSSH algorithm. Instead, following earlier work, ${ }^{59,82}$ the transition probabilities are rescaled to preserve the energy conservation with the following expression:

$$
g_{i j}(t)=\max \left[\tilde{g}_{i j}(t) * b_{i j}(t), 0\right]
$$

with $b_{i j}(t)=e^{-\left(\epsilon_{j}-\epsilon_{i}\right) / \mathrm{k}_{\mathrm{B}} T}$ for $\epsilon_{j}>\epsilon_{i}$, and $b_{i j}(t)=1$ for $\epsilon_{j} \leq \epsilon_{i}$, where $\mathrm{k}_{\mathrm{B}}$ is Boltzmann's constant, and $T$ is the temperature of the system, which is assumed to be constant during the non-adiabatic dynamics. Eq. (23) considers only the positive probability flux. Finally, a switch from the state $\left|\Phi_{i}(\mathbf{r}, \mathbf{R}(t))\right\rangle$ to any other state, $\left|\Phi_{j}(\mathbf{r}, \mathbf{R}(t))\right\rangle$, is accepted only when

$$
\sum_{k=1}^{k=j-1} g_{i k}<\xi \leq \sum_{k=1}^{k=j} g_{i k}
$$

where $\xi$ is a uniform random number between 0 and 1 .

An ensemble of CPA-FSSH trajectories are generated by propagating the nuclei using Eq. (17), by computing the electronic amplitudes using Eq. (15), and by determining the active state using Eq. (24). 


\subsection{Post-processing Tools}

With the generated ensemble of CPA-FSSH trajectories, we compute the adiabatic reduced density matrix as

$$
\bar{\rho}_{i j}(t)=\left\langle\rho_{i j}(t)\right\rangle
$$

where $\langle\ldots\rangle$ represents the ensemble average. The estimator, $\rho_{i j}(t)$, is expressed as

$$
\begin{aligned}
& \rho_{i i}(t)=\left\langle\Phi_{i}(\mathbf{r}, \mathbf{R}(t)) \mid \Phi_{\alpha}(\mathbf{r}, \mathbf{R}(t))\right\rangle=\delta_{i \alpha} \\
& \rho_{i j}(t)=a_{i}^{*}(t) a_{j}(t) \quad(\text { for } \quad i \neq j) .
\end{aligned}
$$

Here, the diagonal elements of $\hat{\rho}$ (i.e., $\left.\rho_{i i}(t)\right)$ are chosen based on the active state $\left|\Phi_{\alpha}(\mathbf{r}, \mathbf{R}(t))\right\rangle$. Along a specific trajectory, $\mathbf{R}(t)$, the instantaneous population at time $t$ is considered as 1 for the active state $\left|\Phi_{\alpha}(\mathbf{r}, \mathbf{R}(t))\right\rangle$, and 0 for all other states. The off-diagonal elements are computed based on the wavefunction expansion coefficients, $a_{j}(t)$.

Apart from the above adiabatic populations, we also need the timedependent diabatic/charge populations, on each moiety of the entire system, to characterize the photo-induced charge transfer dynamics. The charge population on a specific fragment is obtained by projecting the adiabatic reduced density matrix onto the AO basis associated with that molecular fragment, $N$, as:

$$
\left.P_{N}(t)=\operatorname{Re}\left[\sum_{\mu \in N}^{\nu} \sum_{i j} \rho_{i j}(t) C_{\mu i}(t) S_{\mu \nu}(t) C_{\nu j}(t)\right)\right],
$$

where $S_{\mu \nu}(t)=\left\langle\varphi_{\mu}(\mathbf{r}, \mathbf{R}(t)) \mid \varphi_{\nu}(\mathbf{r}, \mathbf{R}(t))\right\rangle$ is the AO overlap matrix at time $t$. The expectation value of the charge population is thus

$$
\bar{P}_{N}(t)=\left\langle P_{N}(t)\right\rangle
$$

where $\langle\ldots\rangle$ represents the ensemble average over the CPA-FSSH trajectories. 


\section{Computational Details}

To describe nonadiabatic electron dynamics in large organic systems, we use the mixed quantum-classical CPA-FSSH method in conjunction with DFTB, which has been shown to give accurate electronic structures for model organic photovoltaic systems ${ }^{83,84}$. The electronic structure calculations are performed at the DFTB3 level of theory ${ }^{72}$ with the 3ob-3-1 Slater-Koster parameter set ${ }^{85}$ as implemented in the DFTB + package ${ }^{57}$. Dispersion interactions between the atoms are incorporated using the Lennard-Jones potential with UFF parameters ${ }^{86}$.

The initialized wavefunction, $|\Psi(\mathbf{r}, 0)\rangle$, is the LUMO of the donor moiety, $\left|\Phi_{\text {LUMO }}^{\mathrm{D}}\right\rangle$, which is a widely used approximation for simulating photoinduced charge transfer dynamics. ${ }^{74,75}$ Here, the LUMO of the donor moiety is obtained from a separate DFTB calculation performed for the isolated donor. From the set of eigenvectors (MOs) of the entire system at the zeroth timestep, $\left\{\left|\Phi_{i}(\mathbf{r} ; \mathbf{R}(\mathbf{0}))\right\rangle\right\}$, an MO maximizing the overlap $\left\langle\Phi_{\mathrm{LUMO}}^{\mathrm{D}} \mid \Phi_{i}(\mathbf{r} ; \mathbf{R})\right\rangle$ is selected.

As explained above, the wavefunction $|\Psi(\mathbf{r}, 0)\rangle$ at the initial time-step is represented as one of the MOs of the entire system. This choice provides a reasonable single-electron picture of the localized photo-excitation in the system. At all other time-steps, to reduce computational cost, the size of the MO basis is truncated to a smaller set containing the LUMO to $\mathrm{LUMO}+9$ orbitals, which are low-lying orbitals that participate directly in the photoinduced charge transfer process.

Several nuclear configurations are then generated with the following procedure. First, the system is equilibrated in an NVT ensemble for 50 ps with a 1 fs nuclear time step using the Nosé-Hoover chain thermostat as implemented in the DFTB+ package. From this NVT trajectory, 30 different nuclear conditions (coordinates and velocities at every 1 ps interval) are collected for the subsequent 4 ps-long quantum dynamics propagation. For

each of these 30 nuclear trajectories, $10^{4} \mathrm{FSSH}$ trajectories should be carried 
out to achieve convergence. To compute the charge transfer population, an ensemble average over both the FSSH and nuclear trajectories is considered.

\section{An Example on Charge Transfer Dynamics in Organic Photovoltaics}

Earlier, we provided an overview of our implementation of mixed quantumclassical CPA-FSSH DFTB for treating CT dynamics. Here, we illustrate the CT dynamics of Phenyl-C61-butyric acid methyl ester/Polythiophene (PCBM-PT), which is a model system for understanding photo-induced charge transfer dynamics in organic photovoltaics. ${ }^{87-90}$

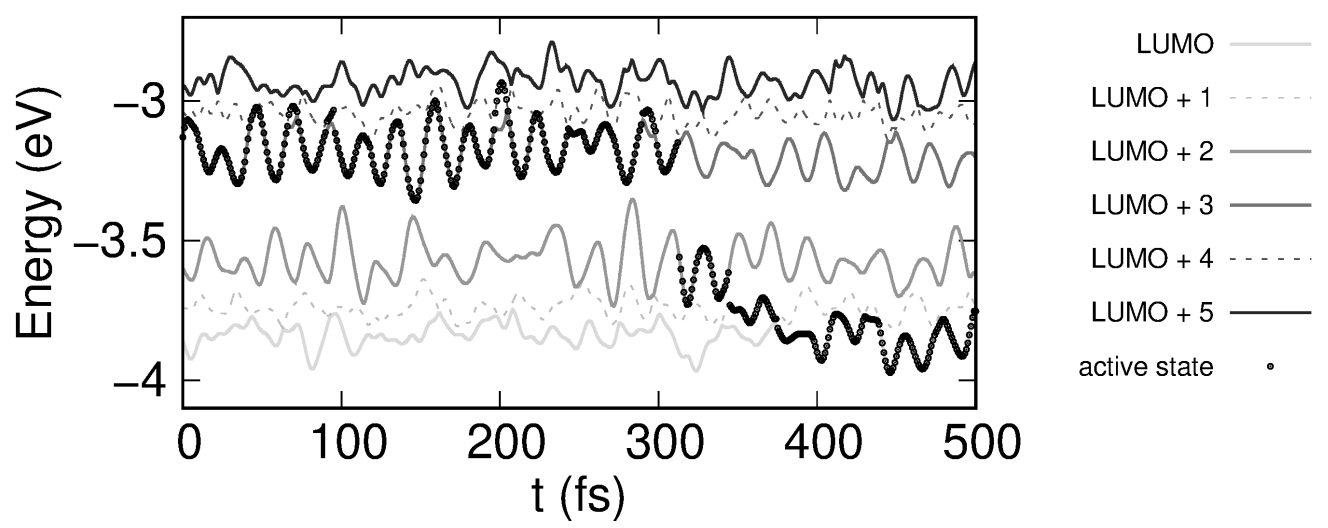

Figure 12: Variations in the energies of the LUMO to $\mathrm{LUMO}+5$ orbitals (adiabatic states) of Phenyl-C61-butyric acid methyl ester/Polythiophene (PCBM-PT) system are shown for a specific nuclear trajectory. Changes in the energy of the active state for a specific FSSH trajectory are also shown. For this FSSH trajectory, the active state switches between the LUMO+3 and LUMO+4 during the first 300 fs of the simulation. Later, it switches to the $\mathrm{LUMO}+2$, the $\mathrm{LUMO}+1$, and finally to the LUMO. Note that, ten thousand FSSH trajectories are considered for each nuclear trajectory.

The simulation begins by initializing the entire system's wavefunction, $|\Psi(\mathbf{r}, 0)\rangle$, by populating the LUMO of the PT molecule, $\left|\Phi_{\mathrm{LUMO}}^{\mathrm{PT}}\right\rangle$, and al- 

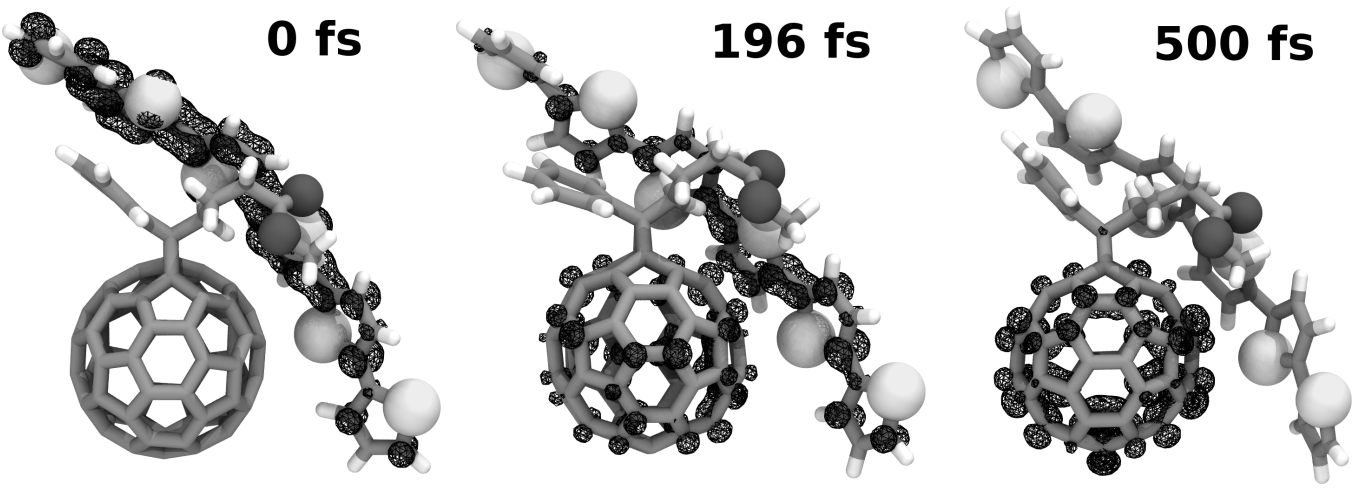

Figure 13: Charge density of the active state in Fig. 12 at various time steps. Carbon and Hydrogen (Oxygen and Sulfur) atoms are represented using gray and white sticks (balls), respectively. The charge density is shown as a black mesh. An iso-value of $0.001 \mathrm{e} /(\text { Bohr })^{3}$ is used.

lowing it to evolve for a few picoseconds according to the CPA-FSSH-DFTB methodology. In Fig. 12 the time-dependent MO energies of the LUMO to LUMO +5 are displayed for a single CPA-FSSH nuclear trajectory during the first 500 fs. Variations in the active state as a function of time for a single FSSH trajectory are also provided in the same panel. At 0 fs, the active state corresponds to the $\mathrm{LUMO}+3$ orbital of the entire system; i.e., $|\Psi(\mathbf{r}, 0)\rangle \equiv\left|\Phi_{\mathrm{LUMO}+3}^{\mathrm{PCBM}-\mathrm{PT}}\right\rangle$, which is primarily localized on the PT molecule $\left(\left|\Phi_{\mathrm{LUMO}+3}^{\mathrm{PCBM}-\mathrm{PT}}\right\rangle \sim\left|\Phi_{\mathrm{LUMO}}^{\mathrm{PT}}\right\rangle\right)$ as shown at the left of Fig. 13. In contrast, the $\mathrm{LUMO}+2$ and $\mathrm{LUMO}+4$ orbitals are localized on the PCBM molecule. As the simulation proceeds, the LUMO +3 continues to be the active state until $\sim 50$ fs. At this stage, the active state hops from the LUMO+3 to the $\mathrm{LUMO}+4$, but quickly returns to the LUMO+3. At the avoided crossings of the $\mathrm{LUMO}+3$ and $\mathrm{LUMO}+4$ ( $\sim 50$ or $190 \mathrm{fs}$ ), we find that both of these orbitals have a mixed character, as shown at the center of Fig. 13. Hops between the LUMO+3 and LUMO+4 are also observed until 300 fs. Thereafter, the active state switches to the low-lying LUMOs (which have a strong PCBM character) and retains its acceptor character until the end of the simulation (as shown at the right of Fig. 13). The hops between the LUMOs 
correspond to the nonadiabatic transitions.
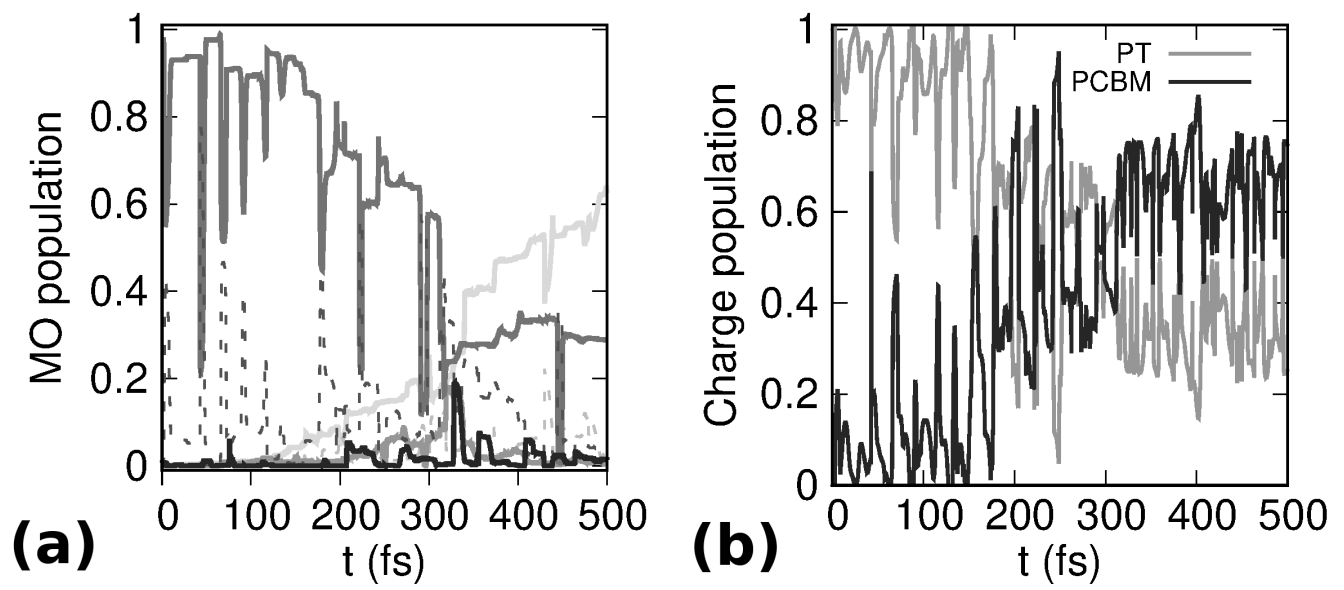

Figure 14: (a) Adiabatic MO and (b) charge population of the PCBM-PT system for a single nuclear trajectory. The same nuclear trajectory as in Fig. 12 is used, but the results are averaged over 10,000 FSSH trajectories. MO colors in (a) are the same as in Fig. 12. Due to the nonadiabatic transitions between the various states, the MO populations change with time. For this nuclear trajectory, an oscillation in the MO population between the $\mathrm{LUMO}+3$ (localized on PT) and $\mathrm{LUMO}+4$ orbitals (localized on PCBM) is observed during the first 100 fs. The same oscillation is also reflected in the charge population plot (panel b), where the gray and black curves represent the charge populations of the PT and PCBM molecules, respectively.

In Figs. 14a and b, we present the adiabatic MO and charge populations calculated for the same nuclear trajectory in Fig. 12, but averaged over 10,000 FSSH trajectories. Due to the nonadiabatic transitions between the various states, the MO populations change with time. However, due to the stochastic nature of the method, these changes are not smooth if we consider only a single CPA-FSSH nuclear trajectory. For example, as shown in Fig. 14a, at $0 \mathrm{fs}$, the MO population is entirely on LUMO+3. However, as shown in Fig. 12, until $\sim 300$ fs, an active state can continuously hop between the LUMO+3 (localized on PT) and LUMO+4 orbitals (localized on PCBM) due to the presence of several avoided crossings between these 
MOs for this nuclear trajectory. Due to these continuous hops, the MO population oscillates, and the same oscillation is also reflected in the charge population (Fig. 14b). Note that, although the same nuclear trajectory is used in obtaining both Figs. 12 and 14a, a one-to-one comparison cannot be made between them. This situation arises because the results in Fig. 14a are averaged over 10,000 FSSH trajectories, whereas the results in Fig. 12 are presented for a single FSSH trajectory. Since the MO energy fluctuations are same for all the 10,000 FSSH trajectories, understanding the nature of a single FSSH trajectory could be useful in understanding the averaged behavior of ten thousand FSSH trajectories. Finally, it is important to note that one cannot accurately assign the charge transfer time-scales using the oscillating charge populations; to obtain any meaningful results, one needs to run at least a few tens of nuclear trajectories.
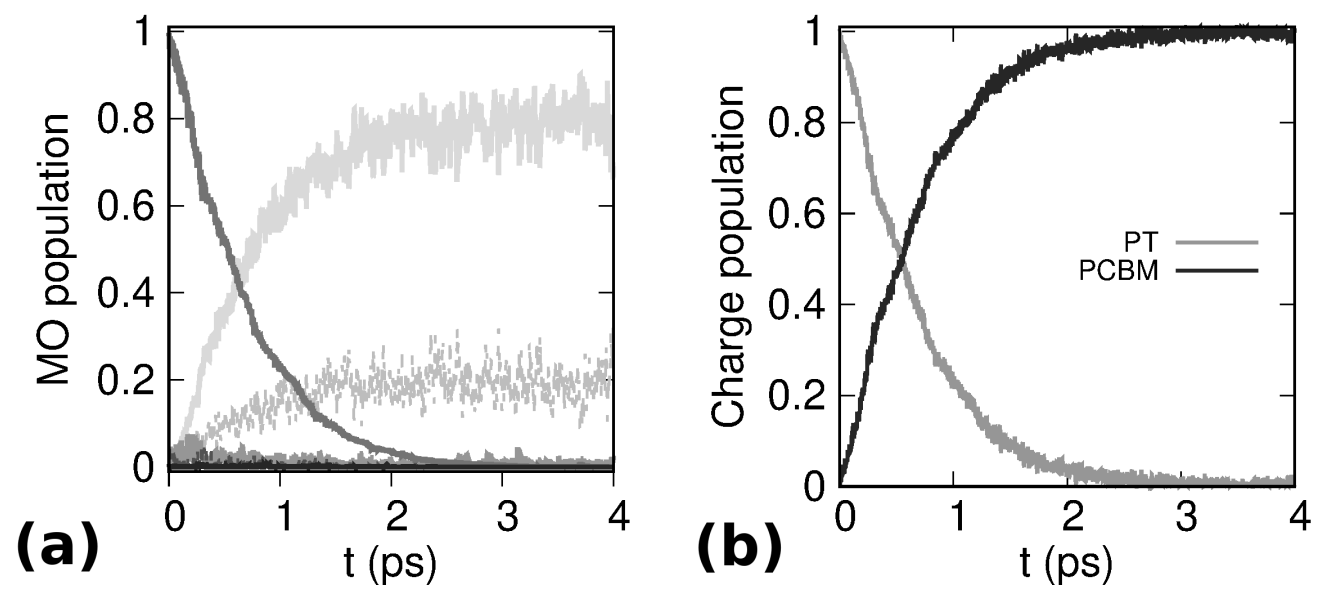

Figure 15: (a) Adiabatic MO and (b) charge population of the PCBM-PT system, averaged over several nuclear and FSSH trajectories. The colors in panel (a) are the same as those used in Fig. 12. The LUMO+3 orbital (localized on PT) loses its population to the LUMO and LUMO+1 orbitals (localized on PCBM), suggesting a charge transfer from the donor to the acceptor. The gray and black curves in panel (b) denote the populations of the PT and PCBM molecules, respectively. 
The MO and charge populations obtained after averaging over $30 \mathrm{nu}-$ clear trajectories and 10,000 FSSH trajectories for each nuclear trajectory are depicted in Fig. 15. In Fig. 15a, the decaying MO population corresponds to the PT-donor molecular orbital (the $\mathrm{LUMO}+3$ ) while the increasing MO populations correspond to the PCBM-acceptor orbitals (the LUMO and LUMO+1). Due to the presence of more than one acceptor orbital, the increasing MO populations continued to exhibit minor oscillations. However, as both of these MOs correspond to the PCBM moiety, such oscillations are not present in the charge populations (recall that the charge populations are obtained by projecting the adiabatic MO populations onto the diabatic AO basis). From the charge populations, we find a complete charge transfer within $\sim 2 \mathrm{ps}$, but most of the population has been transferred by the first picosecond of the simulation. These simulated charge transfer time-scales are in good agreement with earlier ab initio theoretical calculations on similar PCBM-PT models. ${ }^{87-90}$

\section{CONCLUSION AND OUTLOOK}

An overview of DFTB-based excited-state dynamics with applications to both real-time time-dependent DFTB (RT-TDDFTB) and nonadiabatic dynamics has been presented in this review. In both of these formalisms, a series of didactic tutorials and examples demonstrate how each approach is used in practice to reveal dynamical effects in chemical/material systems of contemporary interest. Within the sections on RT-TDDFTB, we demonstrated how this computational approach can be used to either calculate an electronic absorption spectrum or probe the electron dynamics of a chemical system in the presence of monochromatic light. Both of these choices give a different but complementary view of electron dynamics in large sys-

tems, as demonstrated by our example on plasmonic nanoparticles. Within the sections on nonadiabatic dynamics, we explained our implementation 
of CPA-FSSH-DFTB, which was applied to study charge transfer dynamics in an organic photovoltaic system. By computing the charge transfer time-scales in a model PCBM-PT system, which are in good agreement with earlier ab initio results, this approach shows immense promise for probing charge-transfer dynamics in even larger mesoscopic systems. Together, both of these examples in RT-TDDFTB and nonadiabatic dynamics extend the computational efficiency of DFTB to emerging areas of excited-state chemical dynamics, creating an exciting opportunity for understanding these dynamical effects in large, complex systems.

\section{ACKNOWLEDGEMENTS}

The RT-TDDFTB research and applications described in this work were supported by the U.S. Department of Energy, Office of Science, Early Career Research Program under Award No. DE-SC0016269. S. S. R. K. C. Y. acknowledges Prof. Pengfei Huo at the University of Rochester for several helpful discussions related to the CPA-FSSH-DFTB part of the review and for providing computing resources through the Center for Integrated Research Computing (CIRC) at the University of Rochester. M.B.O. acknowledges financial support by the Agencia Nacional de Promoción Científica y Tecnológica (ANPCyT-FONCyT PICT-2017-0795). The authors also acknowledge the Office of Naval Research (grant N00014-18-1-2740) for financial support associated with the extensive research and preparation of this book review.

\section{REFERENCES}

[1] T. Frauenheim, F. Weich, T. Köhler, S. Uhlmann, D. Porezag, and G. Seifert, Phys. Rev. B 52, 11492 (1995). Density-Functional-Based 
Construction of Transferable Nonorthogonal Tight-Binding Potentials for $\mathrm{Si}$ and $\mathrm{SiH}$.

[2] D. Porezag, T. Frauenheim, T. Köhler, G. Seifert, and R. Kaschner, Phys. Rev. B 51, 12947 (1995). Construction of Tight-Binding-Like Potentials on the Basis of Density-Functional Theory: Application to Carbon.

[3] J. Widany, T. Frauenheim, T. Köhler, M. Sternberg, D. Porezag, G. Jungnickel, and G. Seifert, Phys. Rev. B 53, 4443 (1996). DensityFunctional-Based Construction of Transferable Nonorthogonal TightBinding Potentials for B, N, BN, BH, and NH.

[4] M. Elstner, D. Porezag, G. Jungnickel, J. Elsner, M. Haugk, T. Frauenheim, S. Suhai, and G. Seifert, Phys. Rev. B 58, 7260 (1998). SelfConsistent-Charge Density-Functional Tight-Binding Method for Simulations of Complex Materials Properties.

[5] S. I. Allec, Y. Sun, J. Sun, C.-E. a. Chang, and B. M. Wong, J. Chem. Theory Comput. 15, 2807 (2019). Heterogeneous CPU+ GPU-Enabled Simulations for DFTB Molecular Dynamics of Large Chemical and Biological Systems.

[6] S. M. Islam and P.-N. Roy, J. Chem. Theory Comput. 8, 2412 (2012). Performance of the SCC-DFTB Model for Description of Five-Membered Ring Carbohydrate Conformations: Comparison to Force Fields, HighLevel Electronic Structure Methods, and Experiment.

[7] K. Leong, M. E. Foster, B. M. Wong, E. D. Spoerke, D. Van Gough, J. C. Deaton, and M. D. Allendorf, J. Mater. Chem. A 2, 3389 (2014). Energy and Charge Transfer by Donor-Acceptor Pairs Confined in a Metal-Organic Framework: A Spectroscopic and Computational Investigation. 
[8] Q. Cui and M. Elstner, Phys. Chem. Chem. Phys. 16, 14368 (2014). Density Functional Tight Binding: Values of Semi-Empirical Methods in an $\mathrm{Ab}$ Initio Era.

[9] G. Seifert and J.-O. Joswig, WIREs Comput. Mol. Sci. 2, 456 (2012). Density-Functional Tight Binding - An Approximate DensityFunctional Theory Method.

[10] P. Koskinen and V. Mäkinen, Comput. Mater. Sci 47, 237 (2009). Density-Functional Tight-Binding for Beginners.

[11] M. Elstner and G. Seifert, Philos. Trans. R. Soc. A 372, 20120483 (2014). Density Functional Tight Binding.

[12] A. F. Oliveira, G. Seifert, T. Heine, and H. A. A. Duarte, J. Braz. Chem. Soc 20, 1193 (2009). Density-Functional Based Tight-Binding: an Approximate DFT Method.

[13] M. B. Oviedo, X. Zarate, C. F. Negre, E. Schott, R. Arratia-PÉRez, and C. G. SÁNchez, J. Phys. Chem. Lett. 3, 2548 (2012). Quantum Dynamical Simulations as a Tool for Predicting Photoinjection Mechanisms in Dye-Sensitized TiO2 Solar Cells.

[14] C. F. a. Negre, V. C. Fuertes, M. B. Oviedo, F. Y. Oliva, and C. G. SÁNchez, J. Phys. Chem. C 116, 14748 (2012). Quantum Dynamics of Light-Induced Charge Injection in a Model Dye-Nanoparticle Complex.

[15] C. F. A. Negre, K. J. Young, M. B. Oviedo, L. J. Allen, C. G. Sánchez, K. N. Jarzembska, J. B. Benedict, R. H. Crabtree, P. Coppens, G. W. Brudvig, et al., J. Am. Chem. Soc. 136, 16420 (2014). Photoelectrochemical Hole Injection Revealed in Polyoxotitanate Nanocrystals Functionalized with Organic Adsorbates. 
[16] M. B. Oviedo, C. F. A. Negre, and C. G. Sánchez, Phys. Chem. Chem. Phys. 12, 6706 (2010). Dynamical Simulation of the Optical Response of Photosynthetic Pigments.

[17] M. B. Oviedo and C. G. Sánchez, J. Phys. Chem. A 115, 12280 (2011). Transition Dipole Moments of the Qy Band in Photosynthetic Pigments.

[18] C. R. Medrano, M. B. Oviedo, and C. G. Sánchez, Phys. Chem. Chem. Phys. 18, 14840 (2016). Photoinduced Charge-Transfer Dynamics Simulations in Noncovalently Bonded Molecular Aggregates.

[19] C. Mansilla Wettstein, F. P. Bonafé, M. B. Oviedo, and C. G. Sánchez, J. Chem. Phys. 144, 224305 (2016). Optical Properties of Graphene Nanoflakes: Shape Matters.

[20] E. N. Primo, M. B. Oviedo, C. G. Sánchez, M. D. Rubianes, and G. A. Rivas, Bioelectrochemistry 99, 8 (2014). Bioelectrochemical Sensing of Promethazine with Bamboo-Type Multiwalled Carbon Nanotubes Dispersed in Calf-Thymus Double Stranded DNA.

[21] M. B. Oviedo and B. M. Wong, J. Chem. Theory Comput. 12, 1862 (2016). Real-Time Quantum Dynamics Reveals Complex, Many-Body Interactions in Solvated Nanodroplets.

[22] N. V. Ilawe, M. B. Oviedo, and B. M. Wong, J. Chem. Theory Comput. 13, 3442 (2017). Real-Time Quantum Dynamics of Long-Range Electronic Excitation Transfer in Plasmonic Nanoantennas.

[23] N. V. Ilawe, M. B. Oviedo, and B. M. Wong, J. Mater. Chem. C 6, 5857 (2018). Effect of Quantum Tunneling on the Efficiency of Excitation Energy Transfer in Plasmonic Nanoparticle Chain Waveguides.

[24] B. Aradi, B. Hourahine, and T. Frauenheim, J. Phys. Chem. A 111, 5678 (2007). DFTB+, A Sparse Matrix-Based Implementation of the DFTB Method. 
[25] M. Elstner, D. Porezag, G. Jungnickel, J. Elsner, M. Haugk, T. Frauenheim, S. Suhai, and G. Seifert, Phys. Rev. B 58, 7260 (1998). SelfConsistent-Charge Density-Functional Tight-Binding Method for Simulations of Complex Materials Properties.

[26] T. Frauenheim, G. Seifert, M. Elstner, Z. Hajnal, G. Jungnickel, D. Porezag, S. Suhai, and R. Scholz, Phys. Status Solidi B 217, 41 (2000). A Self-Consistent Charge Density-Functional Based TightBinding Method for Predictive Materials Simulations in Physics, Chemistry and Biology.

[27] W. Liang, S. Yokojima, and G. Chen, J. Chem. Phys. 110, 1844 (1999). Generalized Linear-Scaling Localized-Density-Matrix Method.

[28] K. Yabana and G. F. Bertsch, Phys. Rev. B 54, 4484 (1996). TimeDependent Local-Density Approximation in Real Time.

[29] R. G. Parr and R. G. Pearson, J. Am. Chem. Soc. 105, 7512 (1983). Absolute Hardness: Companion Parameter to Absolute Electronegativity.

[30] M. B. Oviedo, Ph.D. thesis, Universidad Nacional De Cordoba, Facultad De Ciencias Químicas, Córdoba, Argentina (2013).

[31] H. Chen, J. M. McMahon, M. A. Ratner, and G. C. Schatz, J. Phys. Chem. C 114, 14384 (2010). Classical Electrodynamics Coupled to Quantum Mechanics for Calculation of Molecular Optical Properties: a RT-TDDFT/FDTD Approach.

[32] D. J. Masiello and G. C. Schatz, J. Chem. Phys. 132, 064102 (2010). On the Linear Response and Scattering of an Interacting Molecule-Metal System.

[33] A. Castro, H. Appel, M. Oliveira, C. a. Rozzi, X. Andrade, F. Lorenzen, M. A. Marques, E. Gross, and A. Rubio, Phys. Status Solidi B 243, 
2465 (2006). Octopus: A Tool for the Application of Time-Dependent Density Functional Theory.

[34] M. BelÉN Oviedo and C. G. SÁNchez, ArXiv E-Prints ArXiv:1502.00491 (2015). Full Quantum Dynamics of the Electronic Coupling Between Photosynthetic Pigments.

[35] A. Boltasseva and H. A. Atwater, Science 331, 290 (2011). Low-Loss Plasmonic Metamaterials.

[36] M. L. Brongersma and V. M. Shalaev, Science 328, 440 (2010). The Case for Plasmonics.

[37] C. M. Soukoulis and M. Wegener, Science 330, 1633 (2010). Optical Metamaterials-More Bulky and Less Lossy.

[38] Y. A. Vlasov, M. O'Boyle, H. F. Hamann, and S. J. McNab, Nature 438, 65 (2005). Active Control of Slow Light on a Chip with Photonic Crystal Waveguides.

[39] D. Solis, A. Paul, J. Olson, L. S. Slaughter, P. Swanglap, W. S. Chang, and S. Link, Nano Lett. 13, 4779 (2013). Turning the Corner: Efficient Energy Transfer in Bent Plasmonic Nanoparticle Chain Waveguides.

[40] M. L. Brongersma, J. W. Hartman, and H. A. Atwater, Phys. Rev. B 62, R16356 (2000). Electromagnetic Energy Transfer and Switching in Nanoparticle Chain Arrays Below the Diffraction Limit.

[41] S. Lal, S. Link, and N. J. Halas, Nat. Photonics 1, 641 (2007). NanoOptics From Sensing to Waveguiding.

[42] G. D. Scholes, G. R. Fleming, A. Olaya-Castro, and R. Van Grondelle, Nat. Chem. 3, 763 (2011). Lessons From Nature About Solar Light Harvesting. 
[43] M. Maiuri, M. B. Oviedo, J. C. Dean, M. Bishop, B. Kudisch, Z. S. D. Toa, B. M. Wong, S. A. McGill, and G. D. Scholes, J. Phys. Chem. Lett. 9, 5548 (2018). High Magnetic Field Detunes Vibronic Resonances in Photosynthetic Light Harvesting.

[44] J. Ma, Z. Wang, and L.-W. Wang, Nat. Commun. 6, 10107 (2015). Interplay Between Plasmon and Single-Particle Excitations in a Metal Nanocluster.

[45] T. LÜNskens, P. Heister, M. ThÄMer, C. A. Walenta, A. Kartouzian, and U. Heiz, Phys. Chem. Chem. Phys. 17, 17541 (2015). Plasmons in Supported Size-Selected Silver Nanoclusters.

[46] M. Quinten, A. Leitner, J. R. Krenn, and F. R. Aussenegg, Opt. Lett. 23, 1331 (1998). Electromagnetic Energy Transport via Linear Chains of Silver Nanoparticles.

[47] B. Willingham and S. Link, Opt. Express 19, 6450 (2011). Energy Transport in Metal Nanoparticle Chains via Sub-Radiant Plasmon Modes.

[48] L. Wu, S. F. Tan, M. Bosman, J. K. Yang, C. A. Nijhuis, and P. Bai, RSC Adv. 6, 70884 (2016). Charge Transfer Plasmon Resonances Across Silver-Molecule-Silver Junctions: Estimating the Terahertz Conductance of Molecules at Near-Infrared Frequencies.

[49] F. Wen, Y. Zhang, S. Gottheim, N. S. King, Y. Zhang, P. Nordlander, and N. J. Halas, ACS Nano 9, 6428 (2015). Charge Transfer Plasmons: Optical Frequency Conductances and Tunable Infrared Resonances.

[50] W. Zhu, R. Esteban, A. G. Borisov, J. J. Baumberg, P. Nordlander, H. J. Lezec, J. Aizpurua, and K. B. Crozier, Nat. Commun. 7, 11495 (2016). Quantum Mechanical Effects in Plasmonic Structures with Subnanometre Gaps. 
[51] L. Wu, H. Duan, P. Bai, M. Bosman, J. K. W. Yang, and E. P. Li, ACS Nano 7, 707 (2012). Fowler-Nordheim Tunneling Induced Charge Transfer Plasmons Between Nearly-Touching Nanoparticles.

[52] J. Hutter, M. Iannuzzi, F. Schiffmann, and J. VandeVondele, WIREs Comput. Mol. Sci. 4, 15 (2014). CP2K: Atomistic Simulations of Condensed Matter Systems.

[53] P. Giannozzi, S. Baroni, N. Bonini, M. Calandra, R. Car, C. Cavazzoni, D. Ceresoli, G. L. Chiarotti, M. Cococcioni, I. Dabo, et al., J. Phys.: Condens. Matter 21, 395502 (2009). QUANTUM ESPRESSO: a Modular and Open-Source Software Project for Quantum Simulations of Materials.

[54] G. Kresse and J. Hafner, Phys. Rev. B 47, 558 (1993). Ab Initio Molecular Dynamics for Liquid Metals.

[55] J. M. Soler, E. Artacho, J. D. Gale, A. García, J. Junquera, P. Ordejón, and D. Sánchez-Portal, J. Phys.: Condens. Matter 14, 2745 (2002). The SIESTA Method for Ab Initio Order-N Materials Simulation.

[56] M. Valiev, E. J. Bylaska, N. Govind, K. Kowalski, T. P. Straatsma, H. J. Van Dam, D. Wang, J. Nieplocha, E. Apra, T. L. Windus, et al., Comput. Phys. Commun. 181, 1477 (2010). NWChem: A Comprehensive and Scalable Open-Source Solution for Large Scale Molecular Simulations.

[57] B. Aradi, B. Hourahine, and T. Frauenheim, J. Phys. Chem. A 111, 5678 (2007). DFTB+, A Sparse Matrix-Based Implementation of the DFTB Method.

[58] Y. Shao, Z. Gan, E. Epifanovsky, A. T. Gilbert, M. Wormit, J. Kussmann, A. W. Lange, A. Behn, J. Deng, X. Feng, et al., Mol. Phys. 113, 
184 (2015). Advances in Molecular Quantum Chemistry Contained in the Q-Chem 4 Program Package.

[59] A. V. Akimov and O. V. Prezhdo, J. Chem. Theory Comput. 9, 4959 (2013). The PYXAID Program for Non-Adiabatic Molecular Dynamics in Condensed Matter Systems.

[60] M. Barbatti, G. Granucci, M. Persico, M. Ruckenbauer, M. Vazdar, M. Eckert-MaksiĆ, and H. Lischka, J. Photochem. Photobiol., A 190, 228 (2007). The On-the-Fly Surface-Hopping Program System NewtonX: Application to Ab Initio Simulation of the Nonadiabatic Photodynamics of Benchmark Systems.

[61] S. Mai, P. Marquetand, and L. González, WIREs Comput. Mol. Sci. 8, E1370 (2018). Nonadiabatic Dynamics: the SHARC Approach.

[62] F. Zapata, L. Ridder, J. Hidding, C. R. Jacob, I. Infante, and L. Visscher, J. Chem. Inf. Model. 59, 3191 (2019). QMflows-A Toolkit for Interoperable Parallel Workflows in Quantum Chemistry.

[63] A. V. Akimov, J. Comput. Chem. 37, 1626 (2016). Libra: An OpenSource "Methodology Discovery" Library for Quantum and Classical Dynamics Simulations.

[64] S. Hammes-Schiffer and J. C. Tully, J. Chem. Phys. 12, 4657 (1994). Proton Transfer in Solution: Molecular Dynamics with Quantum Transitions.

[65] J. C. Tully, J. Chem. Phys. 93, 1061 (1990). Molecular Dynamics with Electronic Transitions.

[66] H. M. Jaeger, S. Fischer, and O. V. Prezhdo, J. Chem. Phys. 137, 22A545 (2012). Decoherence-Induced Surface Hopping. 
[67] N. Shenvi, S. Roy, and J. C. Tully, J. Chem. Phys. 130, 174107 (2009). Nonadiabatic Dynamics at Metal Surfaces: Independent-Electron Surface Hopping.

[68] L. Wang, A. Akimov, and O. V. Prezhdo, J. Phys. Chem. Lett. 7, 2100 (2016). Recent Progress in Surface Hopping: 2011-2015.

[69] M. Barbatti, WIREs Comput. Mol. Sci. 1, 620 (2011). Nonadiabatic Dynamics with Trajectory Surface Hopping Method.

[70] R. Crespo-Otero and M. Barbatti, Chem. Rev. 118, 7026 (2018). Recent Advances and Perspectives on Nonadiabatic Mixed Quantum-Classical Dynamics.

[71] J. E. Subotnik, A. Jain, B. Landry, A. Petit, W. Ouyang, and N. Bellonzi, Annu. Rev. Phys. Chem. 67, 387 (2016). Understanding the Surface Hopping View of Electronic Transitions and Decoherence.

[72] M. Gaus, Q. Cui, and M. Elstner, J. Chem. Theory Comput. 7, 931 (2011). DFTB3: Extension of the Self-Consistent-Charge DensityFunctional Tight-Binding Method (SCC-DFTB).

[73] C. F. Craig, W. R. Duncan, and O. V. Prezhdo, Phys. Rev. Lett. 95, 163001 (2005). Trajectory Surface Hopping in the Time-Dependent Kohn-Sham Approach for Electron-Nuclear Dynamics.

[74] L. G. Rego and V. S. Batista, J. Am. Chem. Soc. 125, 7989 (2003). Quantum Dynamics Simulations of Interfacial Electron Transfer in Sensitized TiO2 Semiconductors.

[75] S. G. Abuabara, L. G. C. Rego, and V. S. Batista, J. Am. Chem. Soc. 127, 18234 (2005). Influence of Thermal Fluctuations on Interfacial Electron Transfer in Functionalized TiO2 Semiconductors. 
[76] S. Pal, D. J. Trivedi, A. V. Akimov, B. Aradi, T. Frauenheim, and O. V. Prezhdo, J. Chem. Theory Comput. 12 (4), 1436-1448 (2016). Nonadiabatic Molecular Dynamics for Thousand Atom Systems: A TightBinding Approach Toward PYXAID.

[77] L. G. Rego, B. C. Hames, K. T. Mazon, and J.-O. Joswig, J. Phys. Chem. C 118, 126 (2013). Intramolecular Polarization Induces Electron-Hole Charge Separation in Light-Harvesting Molecular Triads.

[78] A. Torres, R. S. Oliboni, and L. G. Rego, J. Phys. Chem. Lett. 6, 4927 (2015). Vibronic and Coherent Effects on Interfacial Electron Transfer Dynamics.

[79] B. Space and D. Coker, J. Chem. Phys. 94, 1976 (1991). Nonadiabatic Dynamics of Excited Excess Electrons in Simple Fluids.

[80] N. Yu, C. Margulis, and D. Coker, J. Phys. Chem. B 105, 6728 (2001). Influence of Solvation Environment on Excited State Avoided Crossings and Photodissociation Dynamics.

[81] C. Hu, O. Sugino, H. Hirai, and Y. Tateyama, Phys. Rev. A 82, 062508 (2010). Nonadiabatic Couplings From the Kohn-Sham Derivative Matrix: Formulation by Time-Dependent Density-Functional Theory and Evaluation in the Pseudopotential Framework.

[82] W. R. Duncan, C. F. Craig, and O. V. Prezhdo, J. Am. Chem. Soc. 129, 8528 (2007). Time-Domain Ab Initio Study of Charge Relaxation and Recombination in Dye-Sensitized TiO2.

[83] A. E. Jailaubekov, A. P. Willard, J. R. Tritsch, W.-L. Chan, N. Sai, R. Gearba, L. G. Kaake, K. J. Williams, K. Leung, P. J. Rossky, et al., Nat. Mater. 12, 66 (2013). Hot Charge-Transfer Excitons Set the Time Limit for Charge Separation at Donor/Acceptor Interfaces in Organic Photovoltaics. 
[84] M. Lee, E. Geva, and B. Dunietz, J. Phys. Chem. Lett. 5, 3810 (2014). Donor-To-Donor Vs. Donor-To-Acceptor Interfacial Charge Transfer States in the Phthalocyanine-Fullerene Organic Photovoltaic System.

[85] M. Gaus, A. Goez, and M. Elstner, J. Chem. Theory Comput. 9, 338 (2013). Parametrization and Benchmark of DFTB3 for Organic Molecules.

[86] A. K. Rappe, C. J. Casewit, K. S. Colwell, W. A. Goddard, and W. M. Skiff, J. Am. Chem. Soc. 114, 10024 (1992). UFF, a Full Periodic Table Force Field for Molecular Mechanics and Molecular Dynamics Simulations.

[87] T. Liu and A. Troisi, J. Phys. Chem. C 115, 2406 (2011). Absolute Rate of Charge Separation and Recombination in a Molecular Model of the P3HT/PCBM Interface.

[88] Z.-W. Zhao, Q.-Q. Pan, Y. Geng, Y. Wu, L. Zhao, M. Zhang, and Z.M. Su, ACS Sustain. Chem. Eng. 7, 19699 (2019). Theoretical Insight Into Multiple Charge-Transfer Mechanisms at the P3HT/Nonfullerenes Interface in Organic Solar Cells.

[89] Z. Xu, Y. Zhou, L. Groß, A. De Sio, C. Y. Yam, C. Lienau, T. Frauenheim, and G. Chen, Nano Lett. 19, 8630 (2019). Coherent Real-Space Charge Transport Across a Donor-Acceptor Interface Mediated by Vibronic Couplings.

[90] T. P. Kaloni, P. K. Giesbrecht, G. Schreckenbach, and M. S. Freund, Chem. Mater. 29, 10248 (2017). Polythiophene: From Fundamental Perspectives to Applications. 